\title{
La transformation de l'ancienne Royal High School à Édimbourg, un projet controversé
}

The Transformation of the Old Royal High School in Edinburgh: A Controversial Project

\section{Clarisse Godard Desmarest}

\section{(2) OpenEdition}

\section{Journals}

\section{Édition électronique}

URL : http://journals.openedition.org/etudesecossaises/1365

ISSN : 1969-6337

Éditeur

UGA Éditions/Université Grenoble Alpes

\section{Édition imprimée}

ISBN : 978-2-37747-047-7

ISSN : 1240-1439

Référence électronique

Clarisse Godard Desmarest, « La transformation de l'ancienne Royal High School à Édimbourg, un projet controversé », Études écossaises [En ligne], 20 | 2018, mis en ligne le 01 avril 2018, consulté le 08 septembre 2020. URL : http://journals.openedition.org/etudesecossaises/1365

Ce document a été généré automatiquement le 8 septembre 2020.

(c) Études écossaises 


\section{La transformation de l'ancienne Royal High School à Édimbourg, un projet controversé}

The Transformation of the Old Royal High School in Edinburgh: A Controversial Project

Clarisse Godard Desmarest

1 À Édimbourg, la Royal High School est une institution ancienne fondée il y a environ huit cents ans. Au cours de son histoire, elle a élu domicile à différents endroits de la ville et est située, depuis 1968, à Barnton dans la périphérie nord-ouest de la capitale écossaise. Après un rappel historique, cet article s'intéressera à la signification du bâtiment que l'école a occupé de 1829 à 1968 sur le versant sud de Calton Hill, l'un des promontoires d'Édimbourg (fig. 1). Les projets contemporains de réhabilitation et de transformation de ce bâtiment historique appelé aujourd'hui New Parliament House ou Old Royal High School (5-7 Regent Road) seront ensuite analysés. Cette construction majeure classée "Category A » en 1966 est située dans une zone protégée par les New Town Conservation Area et Old and New Towns of Edinburgh World Heritage Site. Le site fait aussi partie des jardins de la Nouvelle Ville et est répertorié, par Historic Environment Scotland (HES), dans Inventory of Gardens and Designed Landscapes in Scotland (Historic Scotland, 2007).

2 Édifice de style néo-grec conçu par l'architecte Thomas Hamilton (1784-1858), l'ancienne Royal High School a participé au façonnement de l'identité d'Édimbourg en tant qu'" Athènes du Nord». Selon l'historien de l'architecture anglais John Summerson, "[it was] surely the noblest monument of the Scottish Greek Revival » (1953, p. 311). L'historien Howard Colvin résume : «It is admirably composed, impeccably detailed and magnificently situated." (1995, p. 453-455) Les auteurs de A History of Scottish Architecture (1996) soulignent son importance au regard de l'histoire de l'architecture en Europe : «It was on the flanks of Calton Hill that Hamilton designed what was, perhaps, the single most significant monument of Edinburgh Classical Romanticism and one of the setpieces of archaeological Hellenism in Europe: The Royal High School.» (1996, p. 202) 
Construit entre 1825 et 1829 pour répondre à une finalité exclusivement fonctionnelle, celle d'abriter une école, cet édifice s'insère dès l'origine dans un paysage urbain où les monuments commémoratifs occupent une place essentielle. Ceci apparaît très clairement dans une lithographie représentant le Calton Hill de l'artiste Edwin T. Dolby (1852) (fig. 2). Cette construction est importante à de multiples égards. Si la Royal High School appartient à la ville d'Édimbourg, sa signification est en effet nationale. L'Écosse affirme alors sa place dans l'entité britannique et au sein de l'Empire. L'école est aussi l'expression d'une certaine volonté de "démocratie académique » visant à favoriser l'accès du plus grand nombre au savoir. Le choix du Calton Hill pour implanter la Royal High, conséquence d'une nouvelle répartition de la population urbaine, annonce en outre le rôle déterminant que cette colline va jouer pour l'identité d'Édimbourg. Jouxtant le National Monument (1826-1829), réplique inachevée du Parthénon athénien, la Royal High participe à la formation d'un Acropole. Enfin, elle doit être comparée à Edinburgh Academy (fig. 3), une école construite à la même époque dans un style néo-grec et implantée au nord de la Nouvelle Ville, à Stockbridge (fig. 4). Si la Royal High a déménagé depuis sa construction, ce n'est pas le cas de l'Academy qui existe toujours au même endroit.

Depuis le départ de l'école en 1968, les locaux de l'ancienne Royal High ont été peu occupés, ce qui explique que le bâtiment conçu par Thomas Hamilton ait été maintenu dans son intégrité. Les projets actuels soumis à l'analyse de la mairie (propriétaire des lieux), de Historic Environment Scotland et d'Edinburgh World Heritage visent à trouver une nouvelle vocation à cet édifice qui, depuis plusieurs années, a perdu son lustre. La Royal High est au cœur des débats actuels sur la préservation et la restauration des monuments classés à Édimbourg.

\section{Une institution éducative majeure pour la ville d'Édimbourg et pour l'Écosse}

$5 \quad$ L'existence de la Royal High School en tant qu'institution remonte probablement au XII siècle. Les historiens relient volontiers sa fondation à celle de l'abbaye et du monastère de Holyrood. Le roi David $\mathrm{I}^{\mathrm{er}}$ fonda Holyrood en 1128 et il est probable qu'un séminaire, ou institution religieuse, ait vu le jour à la même période à proximité du palais royal. Le document le plus ancien concernant l'école date de 1378 lorsque la présence d'Adam de Camis, responsable de "the grammar schools of the church of Edinburgh", est notée à la faculté de droit de l'université de Montpellier (Barclay, 1974, p. 56-57) ${ }^{1}$. La High School passe sous le contrôle des autorités d'Édimbourg au début du Xvi siècle, sans toutefois que ses liens initiaux privilégiés avec la Couronne ne disparaissent. En 1519, l'école obtient le monopole de l'éducation à Édimbourg et il est fait obligation à tous les bourgeois du bourg d'y placer leur progéniture sous peine de devoir s'acquitter d'une amende (Murray, 1997, p. 1) ${ }^{2}$.

6 Au milieu du XVI ${ }^{e}$ siècle, une bâtisse imposante, illustrée dans Memorials of Edinburgh, est louée au pied de Blackfriars' Wynd (1555-1569) (Wilson, 1848, p. 97, 223 ; Steven, 1849, p.6). Ce bâtiment, accessible par une cour intérieure, avait été le palais ou l'hôtel particulier de l'archevêque James Beaton et de son neveu le cardinal Beaton. À l'époque, Blackfriars' Wynd et les autres ruelles étroites et en pente de la Vieille Ville 
reliant la High Street à Cowgate sont occupés par les citoyens les plus fortunés de la ville.

7 En 1577, grâce à une dotation substantielle de la reine Marie, l'école est relogée par la municipalité dans de nouveaux locaux plus spacieux situés dans le jardin de l'ancien monastère dominicain de Blackfriars, Blackfriars' Yard (devenu par la suite High School Yard). Ce bâtiment, achevé en 1578, constitue la première construction véritablement

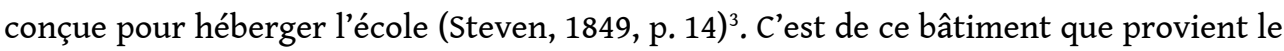
blason sculpté portant la devise de la Royal High School: «Musis Respublica Floret » ("L'État prospère grâce aux Muses») ${ }^{4}$. La Réforme religieuse ne génère pas de mutations profondes pour l'école, mais renforce les liens de celle-ci avec la ville et dissout progressivement ceux avec l'Église. Au cours du XVI e siècle, les chefs successifs de l'établissement, parmi lesquels Hercules Rollock, occupent une place importante dans la vie de la cité (Calderwood, 1842, p. 97)5. Pendant cette période, il est du ressort de la municipalité de veiller au bâtiment de l'école dont elle est propriétaire, de fixer le programme d'étude, d'établir les règles de discipline, de nommer les directeurs et les maitres, de procéder au paiement de ces derniers et de fixer le montant des frais d'inscription. La ville effectue alors plusieurs tentatives pour fonder une université.

8 Sur la carte d'Édimbourg de Gordon of Rothiemay (1647), la proximité géographique entre l'école (Town's School) et l'université (Town's College) apparaît très clairement (fig. 5). Ces deux institutions gérées par la ville sont situées à Kirk of Fields, un beau terrain peu urbanisé, et datent de la même période puisque l'université est fondée en 1583. La légende de la carte mentionne "W. Academia » et «X. Schola Latina », soit l'université et l'école latine. L'enseignement repose alors sur l'apprentissage des lettres classiques et du latin. Le développement de la Royal High School s'effectue en parallèle de celui de l'université.

9 Sur la carte de William Edgar(1765) (fig.6), dans cette même zone du bourg d'Édimbourg, se concentrent l'université (College), l'école (High School) et l'hôpital (Royal Infirmary), ce qui démontre une proximité entre l'enseignement des textes classiques et celui de la médecine. Dans ce dernier champ d'étude, Édimbourg commence à exceller (les examens à la faculté de médecine sont en latin jusqu'en 1826). En 1763, on compte un peu moins de 200 écoliers et pas moins de 500 vingt ans plus tard, ce qui fait de la Royal High School la plus grande école de Grande-Bretagne (Arnot, 1816, p. 512). Grâce à l'acquisition d'un terrain à côté du site de Blackfriars appartenant au Royal Infirmary, l'école peut s'étendre à l'intérieur du mur d'enceinte en $1777^{6}$. CEuvre de l'architecte Alexander Laing, cette nouvelle construction est orientée nordsud alors qu'auparavant le bâtiment était orienté est-ouest (fig. 7 et 8)7.

10 Au cours du XVIII ${ }^{\mathrm{e}}$ siècle, la Royal High School forme les futurs acteurs des Lumières écossaises ${ }^{8}$. L'institution est pleine de vitalité lorsque décède, en 1809 , celui qui l'a présidée pendant quarante ans, Alexander Adam. Sir Walter Scott, Lord Cockburn, Francis Horner, Henry Brougham et Francis Jeffrey ont tous fréquenté ses bancs et, au début du $\mathrm{xIX}^{\mathrm{e}}$ siècle, la Royal High School est considérée comme l'École nationale d'Écosse tant son prestige attire des élèves venus de toutes parts : «[...] a school which may truly be considered not only as Metropolitan but National ${ }^{9}$. " En visite à Édimbourg au printemps 1819, un professeur américain fait l'éloge du système éducatif de la Royal High School: " With such advantages of intellectual and moral instruction, is it surprising that Scotland should have taken such an elevated stand among the nations, for the intelligence, industry, and sobriety of her people? » (Griscom, 1824, p. 223; Barclay, 1974, p. 16) ${ }^{10}$ 
11 Vers 1820, l'élan qui avait donné lieu, en 1777, à la construction d'une école sur Infirmary Street s'exprime à nouveau. Les locaux, jugés suffisamment spacieux à l'origine, sont devenus trop exigus du fait de l'accroissement des effectifs (Murray, 1997, p. 38) ${ }^{11}$. La pression démographique se traduit alors par une augmentation de la superficie totale de la ville. Le North Bridge, ce pont chevauchant le Nor'Loch construit entre 1763 et 1772, est en effet le prélude à la construction, au nord de la Vieille Ville, de plusieurs villes nouvelles répondant aux exigences sanitaires et au mode de vie raffiné des élites ${ }^{12}$.

12 Une nouvelle forme de ségrégation sociale s'exprime et les élites installées sur les artères prestigieuses de Queen Street et de Great King Street rechignent peu à peu à envoyer leurs enfants à l'école dans la Vieille Ville. Le North Bridge et le South Bridge, lequel est construit entre 1786 et 1788, forment une artère encombrée. La High School voit donc ses effectifs décroître tandis que de petites écoles privées dans la Nouvelle Ville captent un contingent croissant d'écoliers (environ 500 garçons au milieu des années 1820). L'effectif global de la High School passe de 887 en 1821 à 601 en $1825^{13}$. La municipalité tarde pourtant à réagir, en raison du mauvais état de ses finances. Les investissements nombreux, en particulier au port de Leith, mènent à la faillite de la ville en 1833.

13 L'école est aussi fragilisée par la création, en 1824, d'une institution rivale, l'Edinburgh Academy ${ }^{14}$. Lord Cockburn, Leonard Horner ${ }^{15}$, Henry Brougham, Francis Jeffrey et Sir Walter Scott, quoiqu'anciens élèves de la Royal High, sont à son origine. Cette nouvelle école privée qui fédère des personnalités éminentes de sensibilité whig et tory, peu de temps avant le Great Reform Act (1832), vise à offrir une solide formation en grec aux écoliers ${ }^{16}$. Introduit à la Royal High en 1772 en dépit des protestations émanant de l'université, le grec occupe une place marginale à la "Schola Latina " au début du XIX siècle. Or, sa maîtrise constitue un prérequis à l'entrée des universités anglaises et à la poursuite de carrières prestigieuses dans l'Empire (East India Company). De nombreuses objections sont pourtant émises à l'encontre de l'Academy. Cette école, aux frais de scolarité plus élevés que ceux de la Royal High School, serait destinée à une élite fortunée, contrariant de ce fait l'essence égalitaire des institutions écossaises : " Schools for the higher orders only, are inconsistent with the genius of our Scottish institutions ${ }^{17}$. " Selon George Davie, la tradition du "democratic intellect» est menacée par l'anglicisation progressive du système éducatif écossais au cours du XIX siècle (Davie, 1961, p. 58) ${ }^{18}$. Les détracteurs de l'Academy soulignent, à l'époque de sa fondation, la nature très anglophile du projet éducatif. L'un des objectifs assumés de l'Academy est, en effet, de pouvoir rivaliser avec les écoles privées anglaises sélectives (public schools) qui disposent alors d'un monopole d'entrée aux prestigieuses universités d'Oxford et de Cambridge ${ }^{19}$.

La structure du bâtiment conçu par l'architecte William Burn (1789-1870) apparaît clairement sur l'Ordnance Survey de $1853^{20}$. Le terrain de Canonmills Park (ou Distillery Park), acheté à l'Hôpital Heriot pour un coût modéré, est situé à la périphérie nord d'Édimbourg dans une zone peu urbanisée mais qui concentre, à proximité de Water of Leith, les industries les plus polluantes de la ville (les tanneries de Silvermills, la distillerie Haig et l'usine gazière de Tanfield). Dès l'origine du projet, le choix de ce site avait été contesté, mais la principale inquiétude, relayée par le Scotsman, réside dans le public concerné par cette nouvelle école : «It is known that one objection, one only of any 
weight, has been urged against this New Academy from the start-that it would assume a Patrician character ${ }^{21}$. "

\section{La Royal High School de l'architecte Thomas Hamilton et la symbolique du Calton Hill}

Face à l'insatisfaction grandissante de la population, la municipalité d'Édimbourg propose la construction d'une école au cœur du nouveau centre de gravité de la ville et l'abandon des locaux d'Infirmary Street. Plusieurs sites sont tour à tour envisagés, en particulier le Mound (et les jardins de Princes Street à côté) et l'Excise Office à Saint Andrew Square ${ }^{22}$. À la même époque, on débat de la construction, à Édimbourg, d'un monument à la patrie (National Monument) dédié aux victimes des guerres napoléoniennes (Gifford, 2014, p.43-83). Il devait faire écho à celui de Londres. Plusieurs sites sont alors envisagés (Princes Street, The Mound, Waterloo Place et Calton Hill) pour ce monument écossais et on hésite alors sur la forme qu'il doit prendre: arc de triomphe, panthéon ou parthénon. L'idée qu'Édimbourg serait une sorte d'Athènes du Nord moderne germe au cours de ce débat des années 1817-1819 (Lowrey, 2001, p. 136-157). Le peintre Hugh William Williams (1773-1829), au retour de son voyage en Grèce, est l'un des premiers à établir un parallèle entre Édimbourg et Athènes ${ }^{23}$. Le révérend Archibald Alison, membre du comité en charge de la construction du National Monument, insiste sur la nécessité pour l'Écosse de disposer de son propre monument afin de pouvoir affirmer sa place au sein de l'Empire britannique $^{24}$. Si Londres est la capitale politique, Édimbourg doit devenir la capitale culturelle et intellectuelle, une nouvelle Athènes quand Londres est Rome. L'école et le National Monument sont tous deux pressentis pour occuper le site du Mound. L'architecte William Henry Playfair (1790-1857) a commencé, en 1822, à construire la Royal Scottish Academy, mais le site demeure dans l'ensemble vierge de toute autre construction ${ }^{25}$.

16 Le Calton Hill est finalement retenu pour accueillir le monument aux grands hommes. L'implantation de la Royal High School sur cette colline, aux côtés d'une réplique du Parthénon, s'inscrit donc dans une même volonté de célébration patriotique, et la construction de ces deux bâtiments publics est concomitante. Bien que le Calton Hill ait été systématiquement écarté au cours des années 1822-1823, la Royal High School figure sur la carte d'Édimbourg de 1823 contenant les projets de construction pour la ville, les «intended improvements» (Wood, 1823) ${ }^{26}$. La construction d'infrastructures (Regent Bridge, Regent Road et London Road) aux abords du Calton Hill favorise un accès majestueux à la ville par le nord et par l'est.

La perception de cette partie de la ville change aussi à la faveur du développement de la Nouvelle Ville jusqu'à Leith (troisième Nouvelle Ville), un projet qui devait couvrir une superficie de 80-120 hectares. En 1813, une compétition organisée par la mairie donne lieu à l'élaboration de trente-deux plans d'urbanisation que huit architectes sont invités à commenter. Le rapport rédigé par William Stark (1770-1813) s'avèrera déterminant dans l'évolution de la perception du paysage urbain. Les idées de Stark, décédé prématurément, seront reprises par son élève William H. Playfair, lequel sort victorieux de la compétition pour l'aménagement du Calton Hill (Stark, 1814) ${ }^{27}$. Influencés par la théorie du pittoresque développée au cours de la seconde moitié du $\mathrm{XVIII}^{\mathrm{e}}$ siècle, Stark et Playfair affirment l'importance de préserver la beauté naturelle du 
site, de valoriser sa topographie et d'intégrer la nature dans le développement urbain ${ }^{28}$. Pour Stark, il est nécessaire de positionner les bâtiments publics (qu'il conçoit au demeurant parfaitement classiques et symétriques) sur des courbes afin qu'ils se dévoilent comme par surprise. Cette esthétique urbaine contraste avec celle ayant guidé la construction de la première Nouvelle Ville, laquelle est traversée par des axes aux larges perspectives ${ }^{29}$. Cette valorisation du pittoresque, influencé par les panoramas de Thomas Barker (1739-1806), trouve écho dans les représentations picturales du Calton Hill du milieu du XIX ${ }^{e}$ siècle, et notamment celles de David Roberts. Le panorama d'Édimbourg de John Menzies (1848) figure bien cette opposition entre une Vieille Ville densément peuplée et dominée par les cheminées des usines à gaz et le site préservé du Calton Hill (fig. 9).

Toute l'importance de la Royal High School à l'échelle locale et nationale est affirmée par la ville qui dispose, en 1825, d'agents chargés de collecter des souscriptions à Édimbourg et à Londres, mais aussi à Calcutta, Madras, Bombay et Kingston en Jamaïque $^{30}$. Dans ce pamphlet de 1825 , l'allusion à l'importance de l'école dans le paysage urbain est claire :

The situation [...] requires also that such an erection should be of an ornamental character, as it will be a conspicuous object from many points, and particularly prominent on entering the town by the splendid approach of the Regent's Road. (p. 2)

19 Conformément à la nouvelle esthétique, Regent Road décrit une courbe d'où se dévoile la Royal High School. Membre fondateur de la Royal Scottish Academy (1826), Thomas Hamilton réalise une construction fonctionnelle et un bâtiment imposant de style néoclassique (néo-grec), sorte de temple du savoir (fig. 10 \& 11) (Walker, 1984) ${ }^{31}$. En son centre, une grande salle ovale domine au rez-de-chaussée. À gauche de celle-ci, se situe la classe du directeur et, dans les pavillons latéraux, quatre autres salles de classe (deux par étage). Une salle de lecture et une bibliothèque figurent à droite de la grande salle. Le portique hexastyle de style néo-grec confère sa majesté à un édifice visible depuis la Vieille Ville et qui renvoie, en contrebas, à la Scottish Academy de Playfair sur le Mound. Thomas Hamilton ne s'était pas rendu en Grèce, mais l'ouvrage de Stuart et Revett, Antiquities of Athens (1762), constitue sa source principale d'inspiration. Les références de Hamilton à Athènes sont nombreuses. Le portique de la Royal High School n'est en effet pas sans rappeler celui du temple d'Héphaïstos sur le site de l'Agora grecque et celui du temple de Thésée ${ }^{32}$. Les colonnades d'ordre dorique font, quant à elles, écho au rez-de-chaussée de la stoa d'Attale (Gifford, McWilliam \& Walker, 1984, p. 440).

20 Avant la construction de monuments significatifs sur le Calton Hill, Lord Cockburn affirmait que le surnom de "Modern Athens " pour Édimbourg était à peine justifié3 ${ }^{33}$. Le bâtiment construit par Hamilton est dès lors très important, car il consolide un paysage symbolique illustré par l'architecte George Meikle Kemp (1840-1846). En répondant au Parthénon (le National Monument), la Royal High School joue le rôle de propylée, lequel marque l'entrée de l'acropole à Athènes.

\section{Les projets actuels de transformation de l'ancienne Royal High School}

21 À la suite du départ de l'école en 1968, plusieurs utilisations furent tour à tour envisagées pour l'édifice de Thomas Hamilton. Entre 1977 et 1980, des travaux furent 
menés, sous l'égide de Property Services Agency, en vue d'y accueillir la future Assemblée d'Écosse, mais c'était sans compter l'échec du référendum sur la dévolution en 1979. La grande salle de l'école aurait dû accueillir l'hémicycle de l'Assemblée (fig. 12). Lors de l'adoption du Scotland Act en 1998 et l'introduction de la dévolution à l'Écosse en 1999, il fut à nouveau question pour la Royal High School d'héberger le nouveau parlement écossais. Le Scotland Office opta pourtant pour la construction d'un bâtiment plus grand et spécialement conçu à cet effet à proximité de Holyrood, dans le Canongate. Le secrétaire d'État à l'Écosse, Donald Dewar, préférait alors un site vierge non associé aux revendications nationalistes. L'école et le Calton Hill avaient en effet été le théâtre de la campagne écossaise pour l'auto-détermination dans les années 1980 au point de constituer « a nationalist shibboleth ${ }^{34}$ » (Black, 2001, p. 84-92). Jim Sillars et le 79 Group avaient même effectué, en guise de protestation, une incursion dans l'old Royal High School en octobre 1981 (Lynch, 2002, p. 267) $)^{35}$. Plus récemment, la High School a hébergé les réunions du Scottish Grand Committee et servi de centre pour travailleurs sociaux tandis qu'une partie du terrain était utilisée comme parking public. En 2001, la mairie envisagea la création d'un musée de la Photographie, pour un coût total estimé à 20 millions de livres, mais ce projet devait tourner court en 2004 avec le désengagement de la loterie nationale, principale source de financement. La question d'une reconversion de l'école en un complexe hôtelier de luxe avec spa et salles de conférence se posa avec acuité à partir de 2009.

$\mathrm{Au}$ terme d'un appel à projets, la mairie offrit, en 2010, la concession du bâtiment au consortium immobilier Duddingston House Properties (DHP) pour un bail de 125 ans. Un espace d'art accessible au public devait être intégré à l'hôtel. En mars 2010, l'hôtelier projetait la construction de deux ailes vitrées imposantes contenant un total de 150 chambres de part et d'autre du bâtiment principal construit par Thomas Hamilton. À mesure que les plans du cabinet Hoskins Architects étaient dévoilés, Historic Scotland (HS), prédécesseur de Historic Environment Scotland (HES), s'inquiétait de la capacité du site à accueillir un bâtiment aussi imposant. Les extensions prévues par l'architecte paraissaient menacer toute la symbolique du Calton Hill et l'élégance de Regent Terrace (William Playfair). Les demandes formelles de permis de construire et d'autorisation de transformation sur un bâtiment historique furent déposées le 31 août 2015 à la mairie d'Édimbourg (références : 15/03989/FUL et 15/03990/LBC).

23 Consulté par le Planning and Strategy Department de la mairie, Historic Scotland (HS) notifia son refus d'entériner le projet soumis par l'hôtelier le 30 septembre $2015^{36}$. Au terme d'un rapport détaillé de trente-six pages, Historic Scotland concluait que les plans ne respectaient ni l'intégrité, ni le site, ni la signification de la Royal High School et qu'ils portaient atteinte au paysage du Calton Hill. Depuis 2014, cet organisme doit être systématiquement consulté par la mairie lorsque des propositions de développement urbain sont susceptibles de porter atteinte à la valeur universelle de ce site qui figure au Patrimoine mondial de l'Organisation des Nations unies pour l'éducation, la science et la culture (UNESCO) sous la dénomination «Old and New Towns World Heritage Site ». Le classement engage en effet les autorités locales à assurer la protection, la conservation et la restauration du site et à en assurer un développement maitrisé. Lors de leur inscription sur la liste du patrimoine mondial en 1995, l'Ancienne et de la Nouvelle Ville d'Édimbourg pouvaient justifier d'une valeur universelle et satisfaisaient aux critères de sélection 2 et 4 de l'UNESCO ${ }^{37}$. 
(ii): The successive planned extensions of the New Town, and the high quality of its architecture, set standards for Scotland and beyond, and exerted a major influence on the development of urban architecture and town planning throughout Europe, in the 18th and 19th centuries.

(iv): The Old and New Towns together form a dramatic reflection of significant changes in European urban planning, from the inward looking, defensive walled medieval city of royal palaces, abbeys and organically developed burgage plots in the Old Town, through the expansive formal Enlightenment planning of the 18th and 19th centuries in the New Town, to the 19th century rediscovery and revival of the Old Town with its adaptation of a distinctive Baronial style of architecture in an urban setting ${ }^{38}$.

Dans le dossier soumis à l'UNESCO, la capitale écossaise se targuait de disposer d'une concentration inédite de bâtiments de style néo-classique en Europe :

The New Town is important for two main reasons: for its uncommonly high concentration of world-class neo-classical buildings and for the amazing size of the area covered with classical ashlar-faced architecture, all consistent to a degree without parallel-and, perhaps crucially, all now surviving virtually intact ${ }^{39}$.

Dans la première feuille de route de gestion du site pour la période 2005-2010, la Royal High School était identifiée comme un bâtiment important, au même titre que Register House (1774, Robert Adam) et la Royal Scottish Academy (1822-1836, W. H. Playfair) ${ }^{40}$. Ce chef-d'œuvre de style néo-grec était jugé comparable aux constructions de Leo von Klenze et de Karl F. Schinkel en Allemagne et à celles de William Wilkins et de Robert Smirke en Angleterre. Dans son rapport de septembre 2015, Historic Scotland regrettait que l'hôtelier n'ait su apprécier l'importance de cette construction au regard de l'histoire de l'architecture. L'organisme public craignait que, du fait des extensions, l'école ne se distingue plus clairement dans le paysage et que l'urbanisation du Calton Hill soit renforcée $e^{41}$. Or, cette colline justifie en partie le classement au patrimoine mondial. Historic Scotland rappelait que l'ancienne Royal High School ne prend sens que par rapport aux autres monuments qui l'entourent, comme le National Monument et le Bridewell, la prison construite par Robert Adam et en partie remplacée par le bâtiment Art-Déco de St Andrew's House (Thomas S. Tait, 1937-1939). La High School dispose actuellement de vues dégagées sur l'ensemble du paysage environnant et suit la forme incurvée de Regent Terrace (fig. 13). Historic Scotland notait aussi que ce projet était susceptible de porter atteinte à d'autres bâtiments classés («Category A ») en particulier St Andrew's House, le Burns Monument et le Nelson Monument ${ }^{42}$.

Une mobilisation importante orchestrée par la Cockburn Association, Architectural Heritage Society of Scotland (AHSS) et Edinburgh World Heritage (EWH ${ }^{43}$ a conduit à ce que plus de 2000 lettres d'objection parviennent à la mairie, avant que celle-ci ne rende publique sa décision (fig. 14). En parallèle, les résidents se regroupaient au sein d'une association, Regent, Royal, Carlton Terraces and Mews Association (RRCTMA), pour faire valoir leurs droits et leurs intérêts.

En décembre 2015, le Planning Committee de la mairie rejoignait la position de Historic Scotland et rejetait, par une courte majorité (une voix supplémentaire contre), le projet de construction d'un hôtel de luxe (pour un coût total estimé à 75 millions de livres sterling) géré par Rosewood, une chaîne d'hôtels cinq étoiles. La mairie paraissait alors pour le moins divisée entre les partisans d'un développement du secteur hôtelier supposé garantir emplois et rentrées fiscales et ceux pour lesquels la préservation d'un paysage urbain inédit constituait une condition au dynamisme du secteur touristique. Ces arguments étaient relayés, au sein de la mairie, par deux départements distincts : le Planning Committee et l'Economy Committee. Cette tension entre intérêts 
potentiellement divergents fut exprimée par Alison Johnstone, député au parlement écossais pour le Lothian et membre du parti des Verts, lors d'une longue consultation publique menée à la mairie d'Édimbourg en décembre 2015: «I'm so pleased that members of the planning committee were not seduced by arguments about economic benefit, instead recognising that much of our visitor economy is based on the carefully cherished landscape of our city: an inheritance which this development would have squandered ${ }^{44}$." Lors de cette même consultation, Gordon Dewar, directeur de l'aéroport d'Édimbourg, faisait valoir l'importance du tourisme de luxe pour le développement économique de la ville, et arguait qu'une bouteille de whisky à 10000 livres était vendue chaque semaine en duty free. La clientèle d'affaires venue de Chine et d'Amérique du Nord était ciblée par les acteurs du tourisme.

Parallèlement, l'UNESCO à Paris (Division du Patrimoine) faisait savoir, par la voie de sa directrice, qu'il condamnait le projet de transformation de la Royal High School ${ }^{45}$. L'Organisation demandait à la mairie d'Édimbourg et aux autres acteurs de la conservation des monuments historiques (HES et EWH) de bien vouloir prendre toutes les mesures nécessaires à la sauvegarde de la "valeur universelle exceptionnelle » des Vieille et Nouvelle Villes d'Édimbourg ${ }^{46}$. Le rapport des experts d'ICOMOS-UK, l'antenne britannique de ICOMOS (International Council on Monuments and Sites), rédigé à la suite d'une visite de prospection à Édimbourg (13-14 octobre 2015), était alors transmis au Department of Culture, Media and Sport (DCMS) à Londres ${ }^{47}$. Il décrivait une situation inquiétante :

Regrettably the current situation, including both approved and proposed developments, appears to have tipped the balance inappropriately, away from conservation, towards inappropriate development. There is therefore an urgent need to reconsider, revise and reframe current approaches to development, including conservation and adaptation of existing structures and construction of new structures, so as to deliver greater focus on values, to incorporate appropriate expertise and to improve engagement with the community. (ICOMOS, 2015)

La polémique au sujet de la Royal High School intervient alors que plusieurs autres développements urbains suscitent les craintes des experts ICOMOS. On peut citer la transformation du site de Caltongate, la reconstruction du St James Centre, l'extension de la gare de Waverley, la reconversion de l'ancienne Donaldson's School (West Coates) et celle du India Buildings (11-15 Victoria Street, 18-20 Cowgate ${ }^{48}$. L'UNESCO a émis plusieurs mises en garde, lesquelles pourraient menacer le classement de la Vieille Ville et de la Nouvelle Ville d'Édimbourg au patrimoine mondial.

$\mathrm{Au}$ terme de plusieurs tentatives de conciliation infructueuses menées entre des représentants de la mairie, de Historic Environment Scotland et du promoteur immobilier en février 2016, DHP décidait de faire appel de la décision de la mairie d'Édimbourg (refus de validation du permis de construire et de transformation des lieux) le 17 mars 2016. Cet appel sera jugé par le Directorate for Planning and Environmental Appeals (DPEA), lequel est placé sous l'autorité directe du gouvernement écossais ${ }^{49}$. Cette procédure quasi-judiciaire dépasse désormais l'échelon municipal.

31 En août 2016, avant même que les auditions ne commencent, DHP a sollicité une suspension de la procédure pour pouvoir soumettre un nouveau projet pour un hôtel de taille plus réduite. Le changement de contexte économique et les répercussions du Brexit étaient officiellement invoqués par le promoteur pour justifier ce revirement. Selon certains observateurs, il s'agissait cependant de contrer un projet alternatif 
soumis à la mairie le 10 décembre 2015 et qui vise à accueillir, au sein de la Royal High School, les nouveaux locaux de l'école de musique Saint Mary (références : 15/05662/ FUL-15/05665/LBC). Le permis de construire pour ce projet qui, selon Edinburgh World Heritage, préserve la «valeur universelle du site » a été accordé à l'unanimité par la municipalité d'Édimbourg le 27 février $2017^{50}$. Ce projet concurrentiel, piloté par le Royal High School Preservation Trust (RHSPT) et entièrement financé par une philanthrope américaine, Carol Hogel, ne pourra cependant pas voir le jour tant que la mairie d'Édimbourg demeure tenue, par des clauses contractuelles, de traiter exclusivement avec DHP (échéance du contrat prévue en 2022) ${ }^{51}$.

Actuellement située dans la partie ouest d'Édimbourg, Saint Mary's Music School constitue l'une des principales écoles de musique en Écosse et est rattachée à la cathédrale épiscopale Saint Mary. Le projet de transfert au sein du bâtiment conçu par Hamilton lui permettrait d'accroître ses effectifs et de devenir l'école nationale de musique. Ce projet est soutenu par une partie de la population qui souhaite que l'édifice renoue avec sa vocation initiale de formation intellectuelle. Les résidents du Calton Hill n'ont pas émis d'opposition à ce projet soutenu par la Cockburn Association ${ }^{52}$. Le plan proposé par les cabinets Richard Murphy Architects et Simpson \& Brown, qui furent choisis par la mairie en septembre 2015, apparaît respectueux du site du Calton Hill et comporte trois innovations principales. Il propose la création, à l'est du bâtiment principal, d'espaces de répétition et d'hébergement et, à l'ouest, d'un jardin paysagé accessible au public ainsi que la constitution d'un foyer en sous-sol ouvert sur l'extérieur par une porte vitrée et donnant accès, par l'intérieur, à la salle de concert au- dessus (la grande salle de Hamilton).

Dans l'attente d'une prise de décision consensuelle, l'old Royal High School demeure vide et non chauffée. Ce bâtiment, inscrit depuis février 2009 dans le Buildings at Risk Register for Scotland (liste établie par Historic Environment Scotland), risque de se détériorer encore davantage. Historic Environment Scotland et Edinburgh World Heritage s'accordent sur la nécessité de faire évoluer l'actuel statut quo et de trouver une finalité économiquement viable à la Royal High School afin de sauvegarder ce bâtiment. À l'heure actuelle, le premier projet soumis par DHP n'a pas été retiré et une procédure d'appel est en cours. Par ailleurs, le second projet (références : 17/00588/ FUL-17/00587/LBC) soumis par DHP \& Urbanist Hotels en février 2017 pour un hôtel de 127 chambres a été rejeté à l'unanimité par la mairie (Development Management SubCommittee) le 31 août 2017. La mobilisation de la population et le renouvellement partiel de l'équipe municipale, à la faveur des élections municipales du 4 mai 2017 à Édimbourg, se sont probablement avérés déterminants. La mairie s'est conformée à l'avis négatif rendu par Historic Environment Scotland le 24 mars 2017. Celui-ci reprenait l'essentiel de l'argumentaire de septembre 2015. Edinburgh World Heritage, par la voix de son directeur, faisait également savoir son opposition et soulignait que les changements proposés par le promoteur ne répondaient aucunement aux critiques qui lui avaient été soumises antérieurement ${ }^{53}$. Pourtant, l'hôtelier a décidé de faire appel de cette décision en septembre 2017. Le Directorate for Planning and Environmental Appeals (DPEA) a donc été à nouveau saisi par le gouvernement écossais à qui il devra remettre un rapport sur les deux appels. Le 8 février 2018, un «preexamination meeting " avait lieu à Édimbourg, en présence des deux rapporteurs et des représentants des différentes parties impliquées (DHP \& Urbanist Hotels, mairie d'Édimbourg, HES, Cockburn Association, AHSS, RHSPT, RRCTMA). Au terme de cette consultation, il a été décidé que cette procédure prendrait la forme d'un contradictoire 
«enquiry " plutôt que d'un « hearing », le calendrier : deux sessions en septembre et en octobre 2018.

Pour conclure, la Royal High School se situe au cœur d'un paysage symbolique important pour l'identité d'Édimbourg. La capitale écossaise ambitionne de rivaliser avec Londres sur le plan intellectuel au début du XIX ${ }^{e}$ siècle. Elle souhaite alors incarner l'Athènes du Nord. Aujourd'hui, le bâtiment doit trouver une vocation nouvelle et susciter l'adhésion de la population. Celle-ci a été consultée à l'été 2016 dans le cadre de l'élaboration de la prochaine feuille de route (Management Plan) détaillant le mode de gestion du site Old and New Towns of Edinburgh pour la période 2017-2022 ${ }^{54}$. Ce texte détaillant la vision de la ville à échéance 2050 a été adopté en conseil municipal en septembre $2017^{55}$. La mairie d'Édimbourg, Historic Environment Scotland et Edinburgh World Heritage assuraient prendre toute la mesure des critiques formulées par l'UNESCO et œuvrer de concert à l'élaboration d'une stratégie plus efficace de conservation des monuments historiques, l'objectif étant de se prémunir d'un possible déclassement. En 2011, le gouvernement écossais estimait ainsi que le classement au patrimoine mondial constituait l'un des six atouts majeurs d'Édimbourg ${ }^{56}$. À titre comparatif, Liverpool est inscrite depuis 2004 sur la liste du patrimoine mondial, mais est aujourd'hui menacée de perdre ce statut. Elle figure depuis 2012 sur la liste du patrimoine mondial en péril, au même titre que cinquante-trois autres biens. Dès lors, dans un contexte où Édimbourg souhaite légitimement exploiter son potentiel touristique, on ne peut qu'espérer une gestion raisonnée de cette ressource. Édimbourg a remporté, au printemps 2017, un appel à projet du programme de développement régional européen Interreg visant à promouvoir la conservation des centres urbains historiques. La capitale écossaise sera associée, dans ce cadre, à Porto, Saint-Jacquesde-Compostelle, Bordeaux et Florence ${ }^{57}$.

Figure 1. - Vue de Calton Hill depuis Arthur's Seat, au sud.

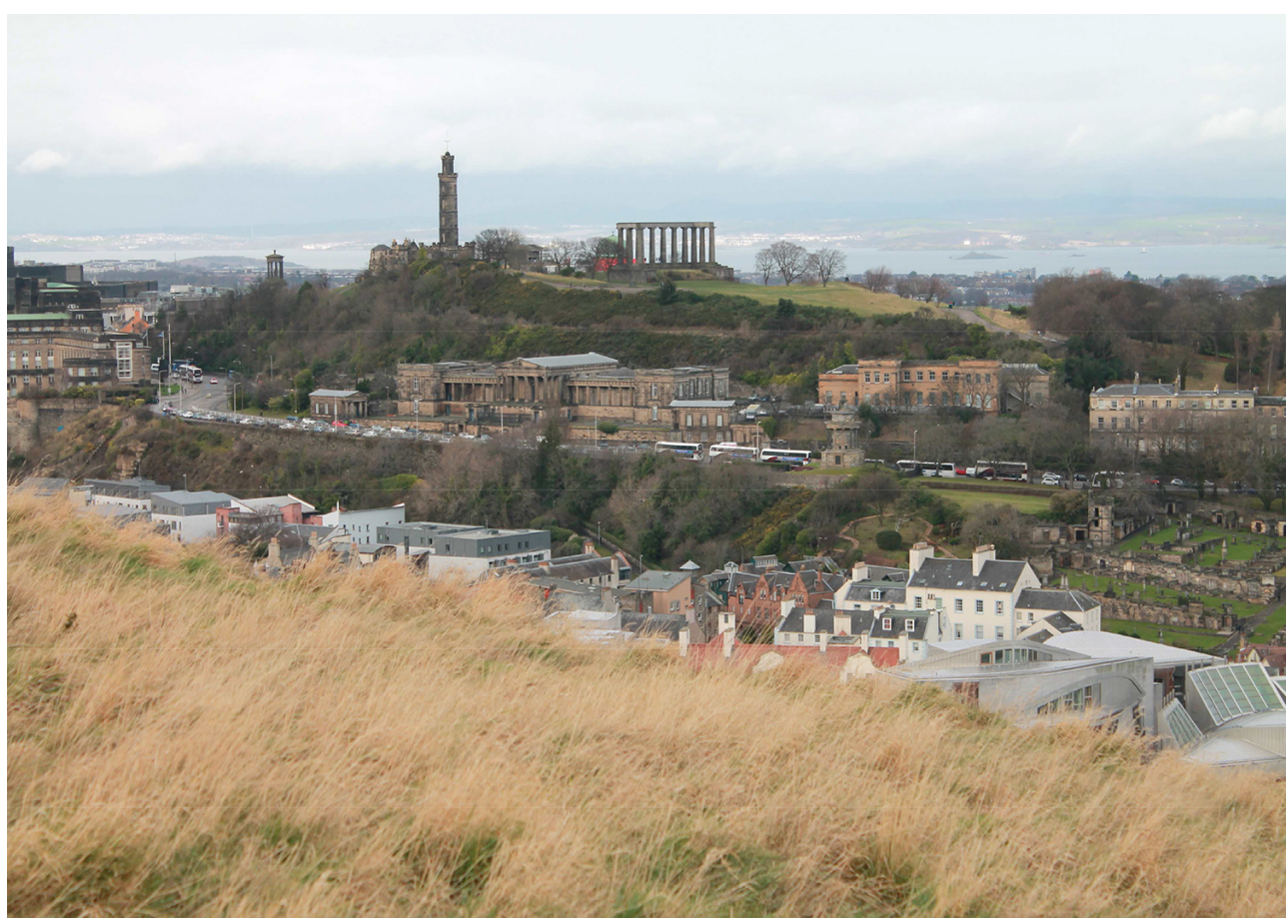

Photographie personnelle, mars 2017. 
Figure 2. - Vue aérienne d'Édimbourg, lithographie d'Edwin T. Dolby, 1852.

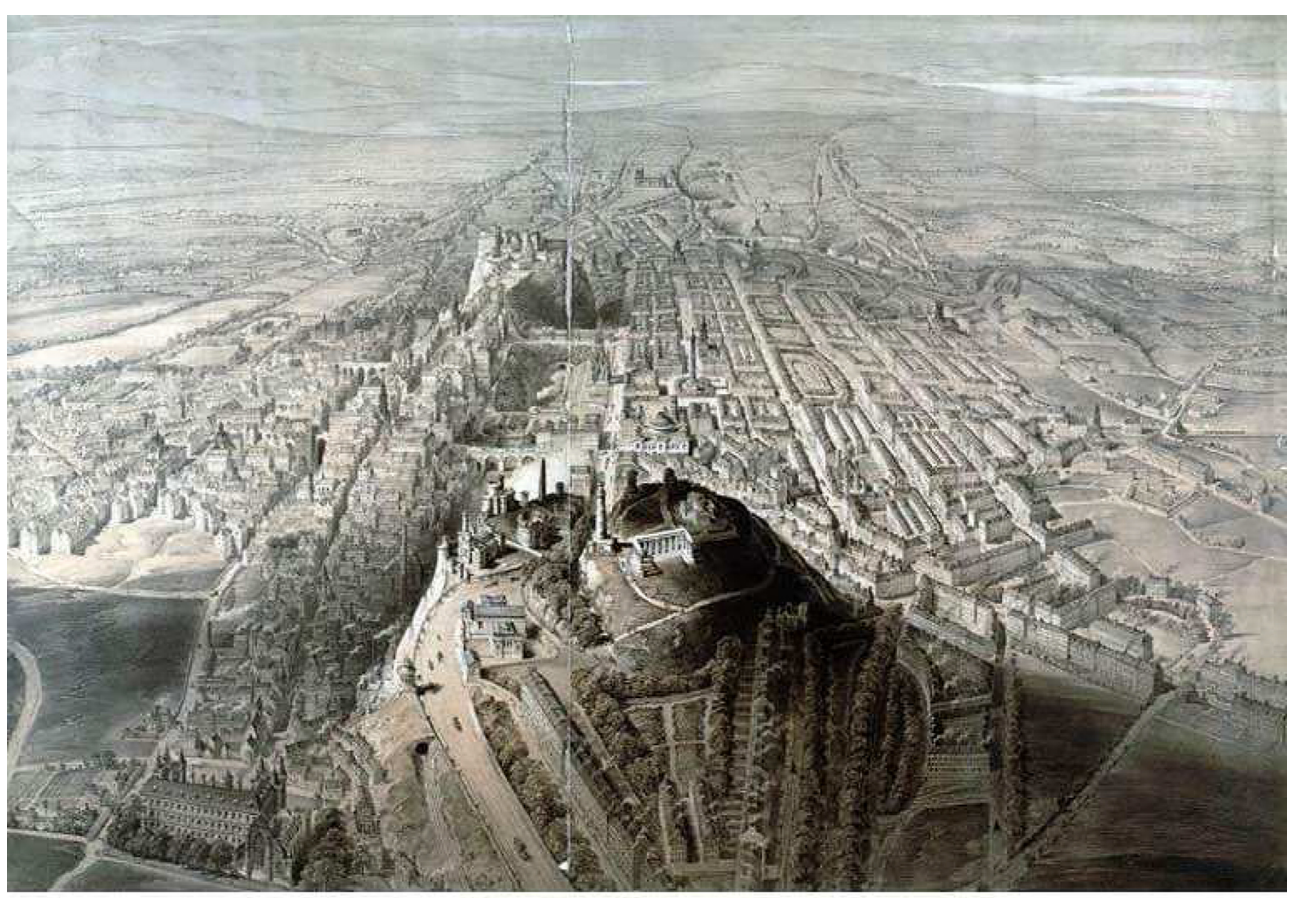

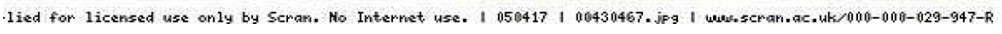

Historic Environment Scotland.

Figure 3. - Edinburgh Academy, architecte William Burn, 1824.

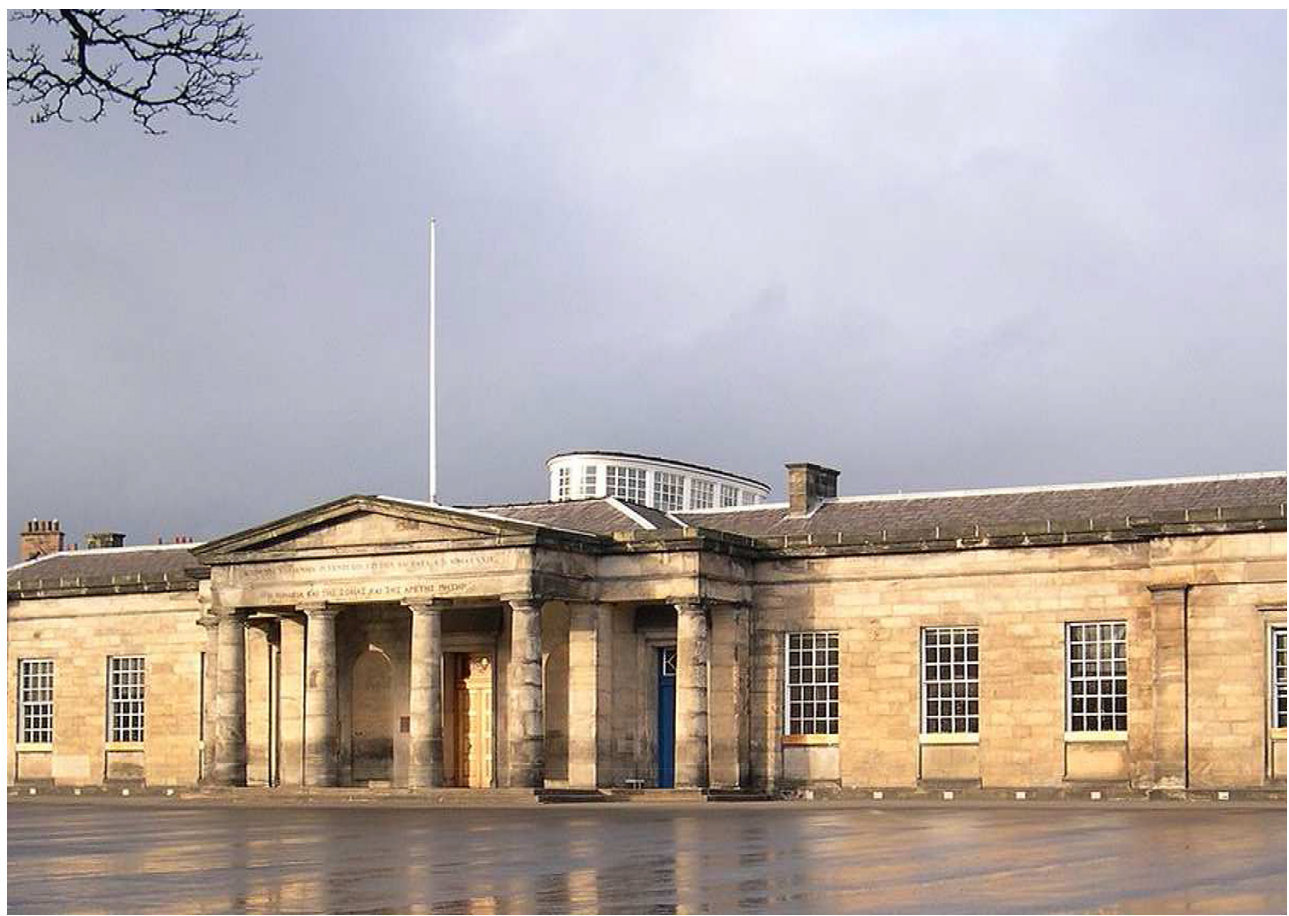

Photographie personnelle, mars 2017 
Figure 4. - Great Reform Act Map of Edinburgh, Londres, Chambre des Communes, 1832.

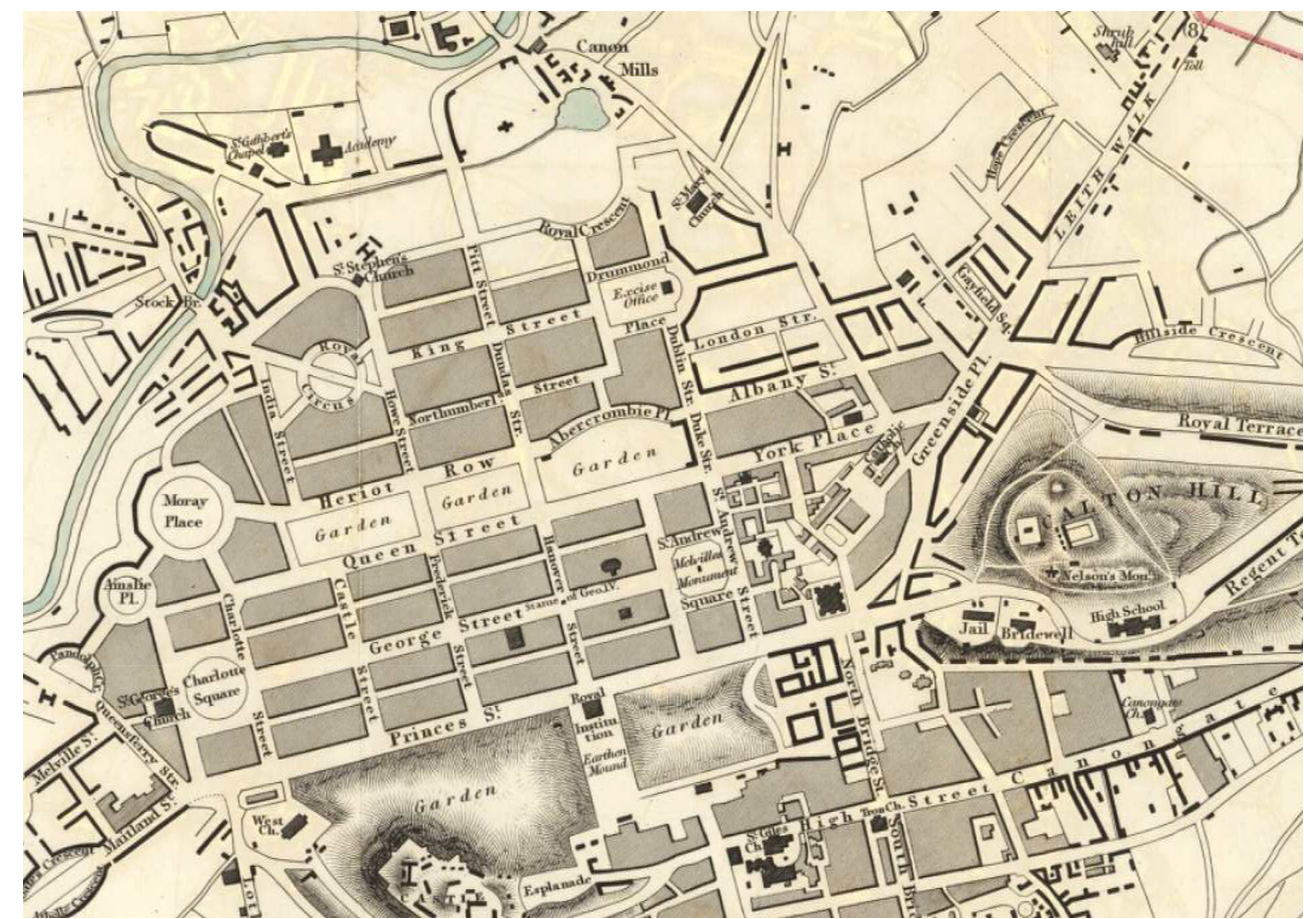

National Library of Scotland.

Figure 5. - Plan d'Édimbourg de James Gordon of Rothiemay, 1647, Édimbourg, W. \& A. K. Johnston, c. 1870.

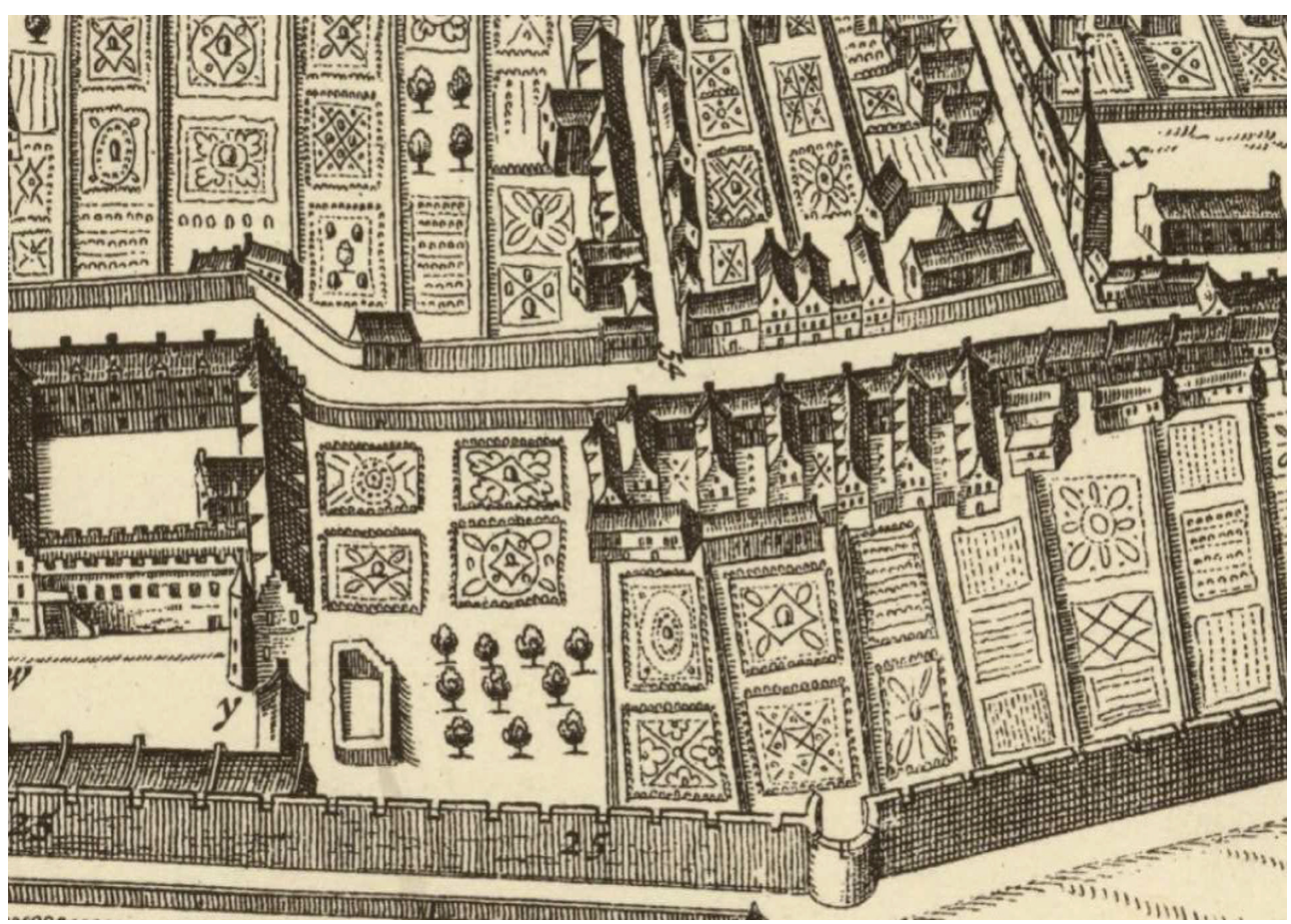

National Library of Scotland. 
Figure 6. - Plan d'Édimbourg de William Edgar, 1765.

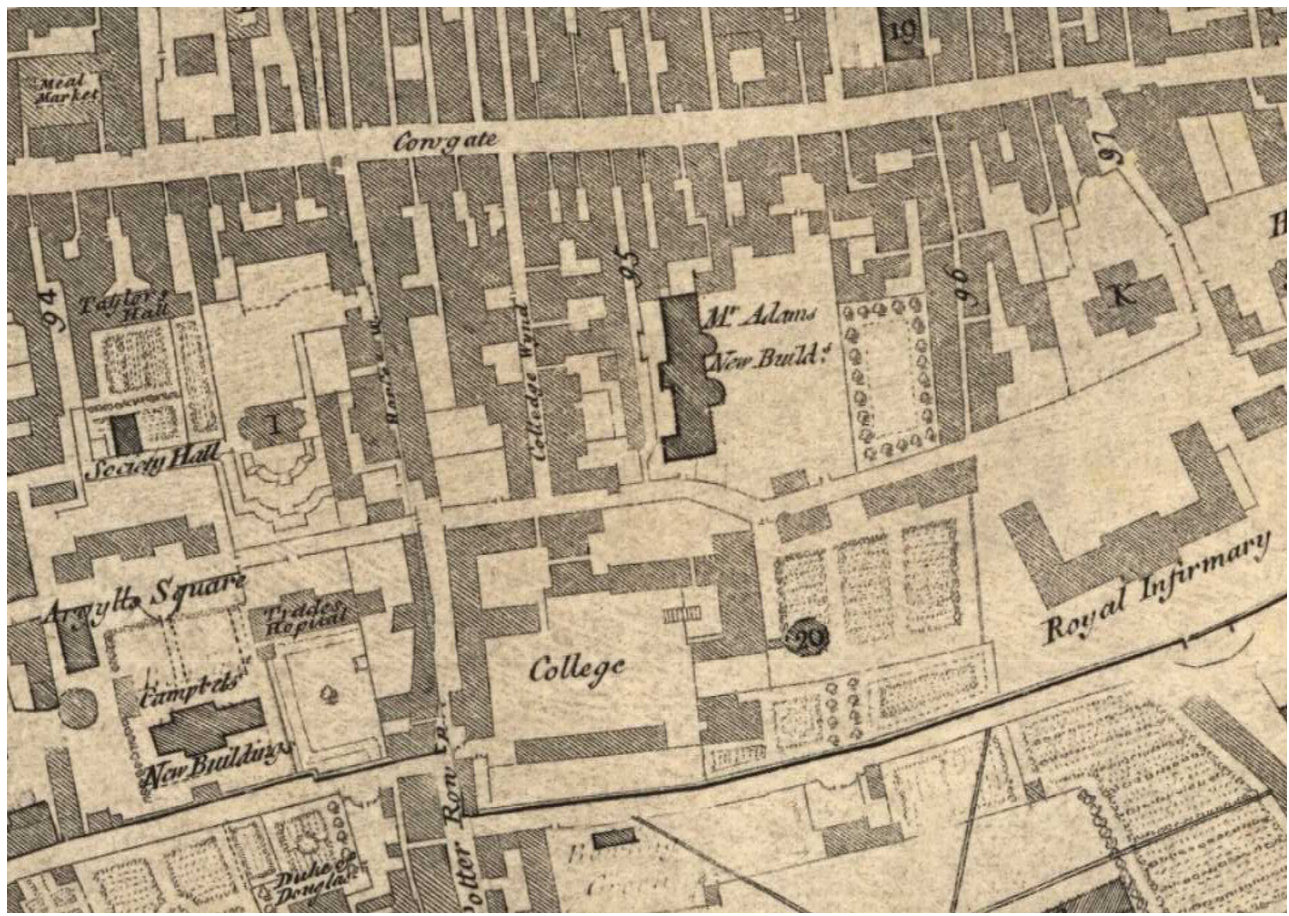

National Library of Scotland.

Figure 7. - Old Royal High School, High School Yard, architecte Alexander Laing, 1777.

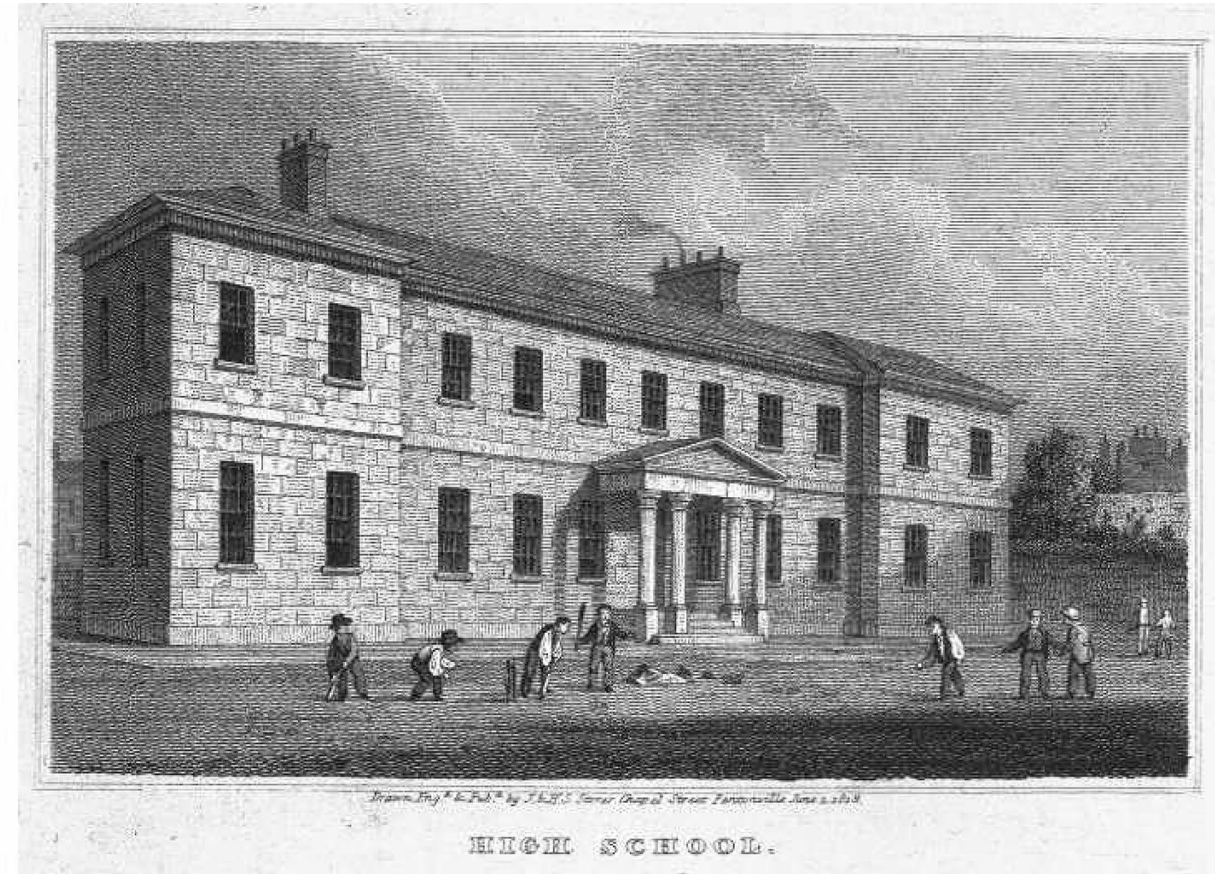

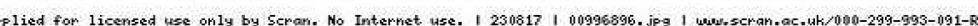

Gravure reproduite dans J. \& H. S. Storer, Views in Edinburgh and Its Vicinity, vol. 2, Édimbourg, Constable, 1820, p. 31 
Figure 8. - Old and New Town of Edinburgh and Leith with the Proposed Docks, Édimbourg, John Ainslie, 1804.

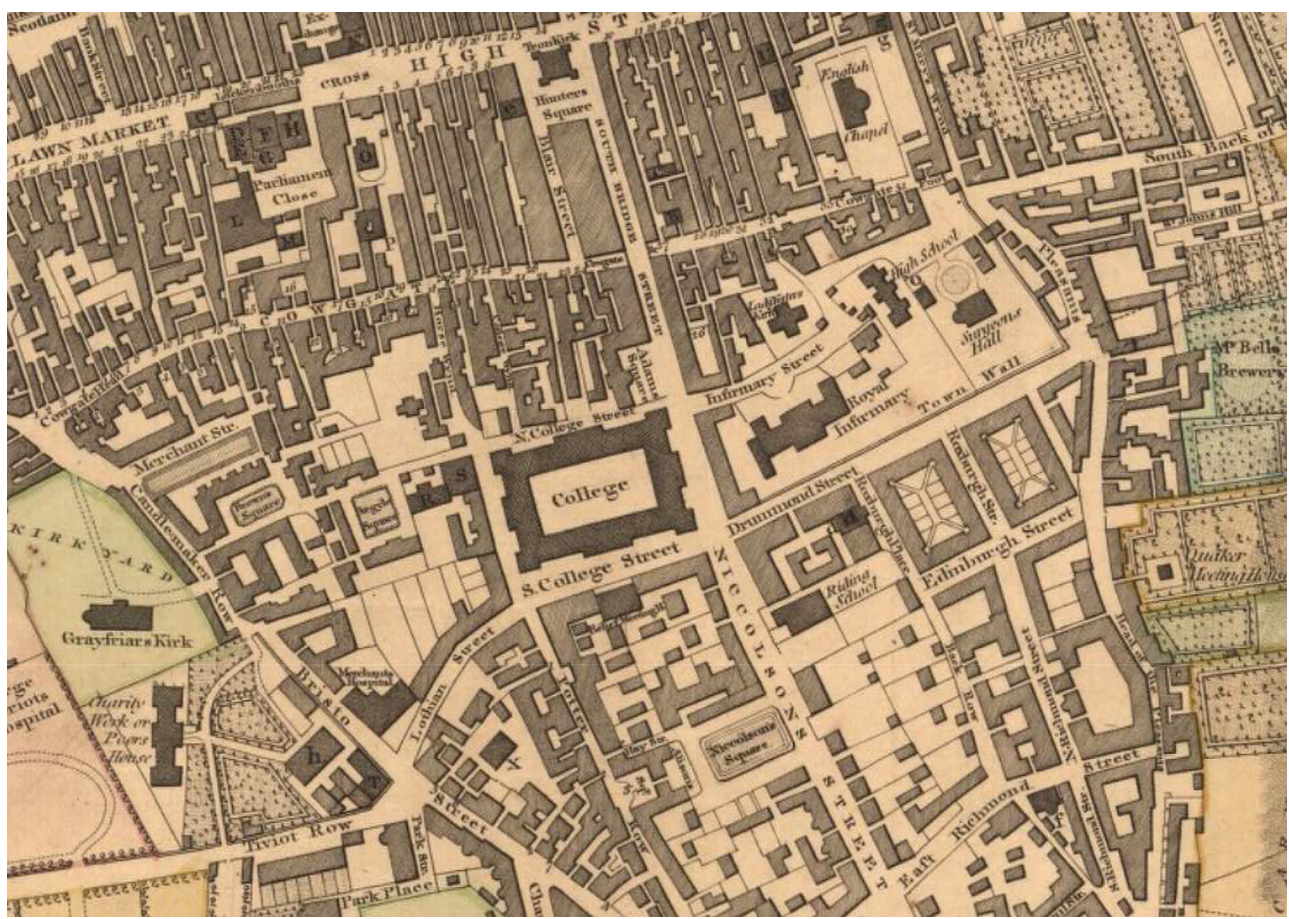

National Library of Scotland.

Figure 9. - John Menzies, Panorama d'Édimbourg vu du Calton Hill, section 3 d'un panorama à $360^{\circ}, 1848$.

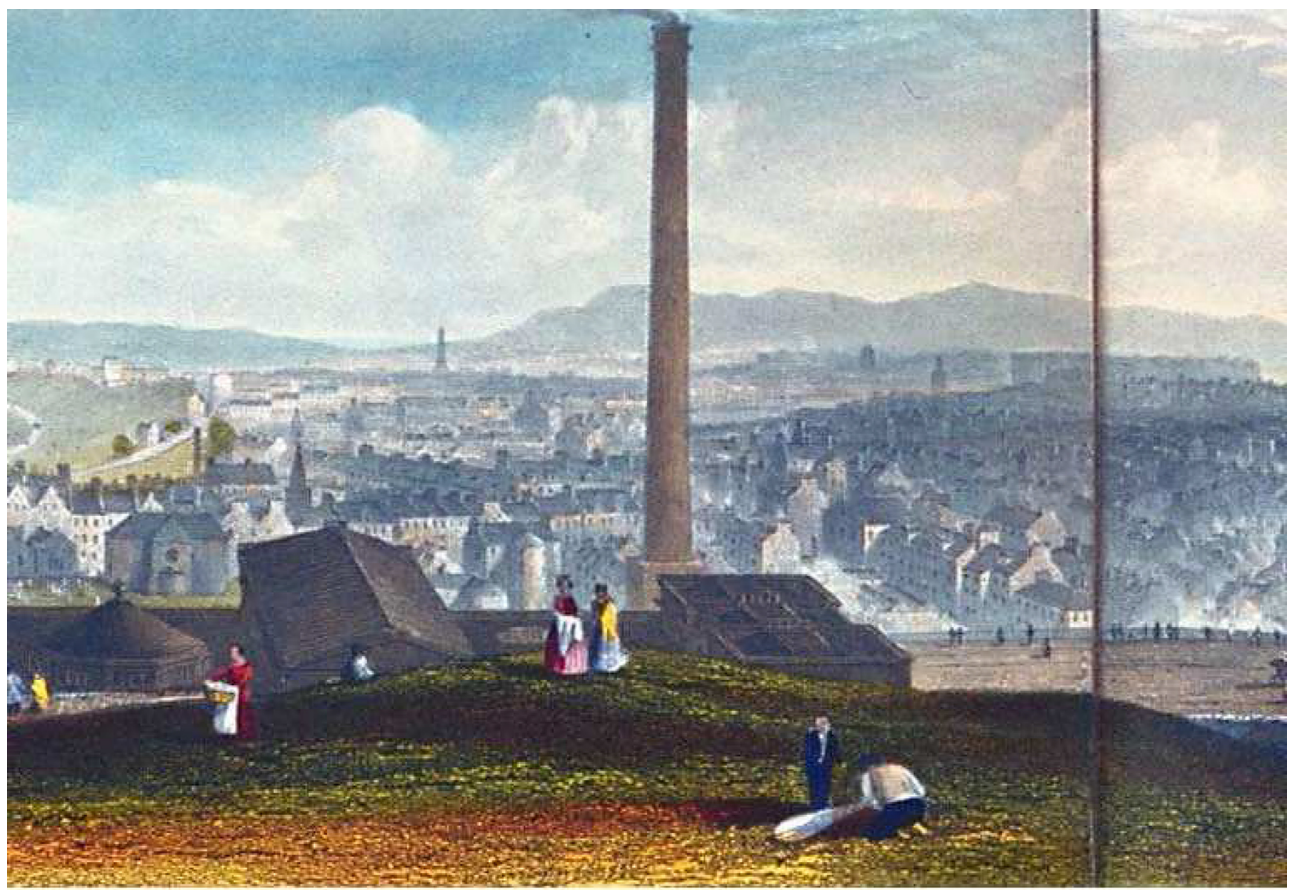

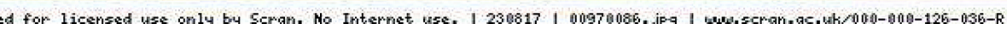

Historic Environment Scotland.

On distingue, au premier plan, le toit de l'ancienne Royal High School de l'architecte Thomas Hamilton et, au second plan, la cheminée d'une usine à gaz. Les Pentland Hills figurent en arrière-plan. 
Figure 10. - Plan de l'Old Royal High School, dessin de l'architecte Thomas Hamilton, 5 octobre 1826.

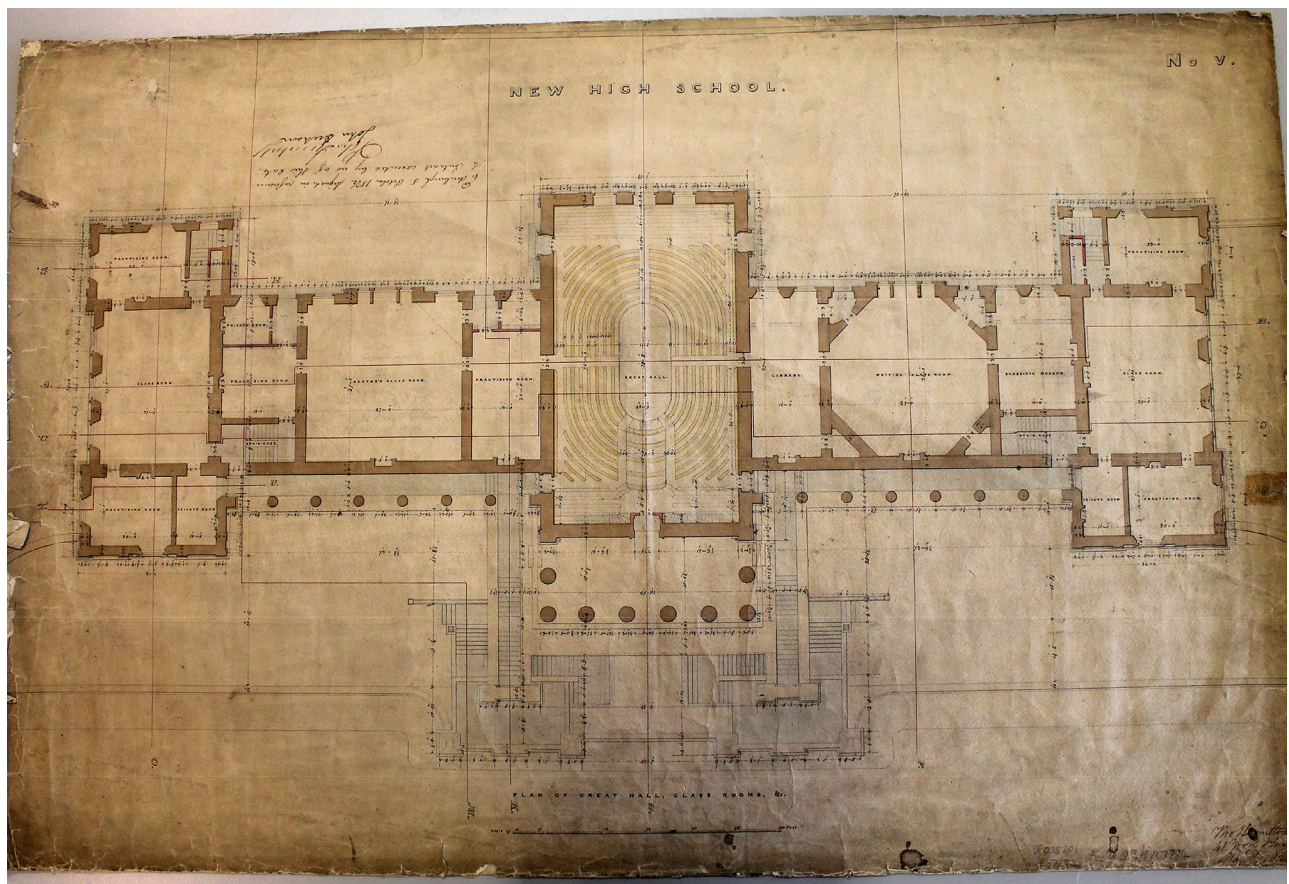

Historic Environment Scotland.

Figure 11. - Façade de l'Old Royal High School, dessin de l'architecte Thomas Hamilton, 5 octobre 1826.

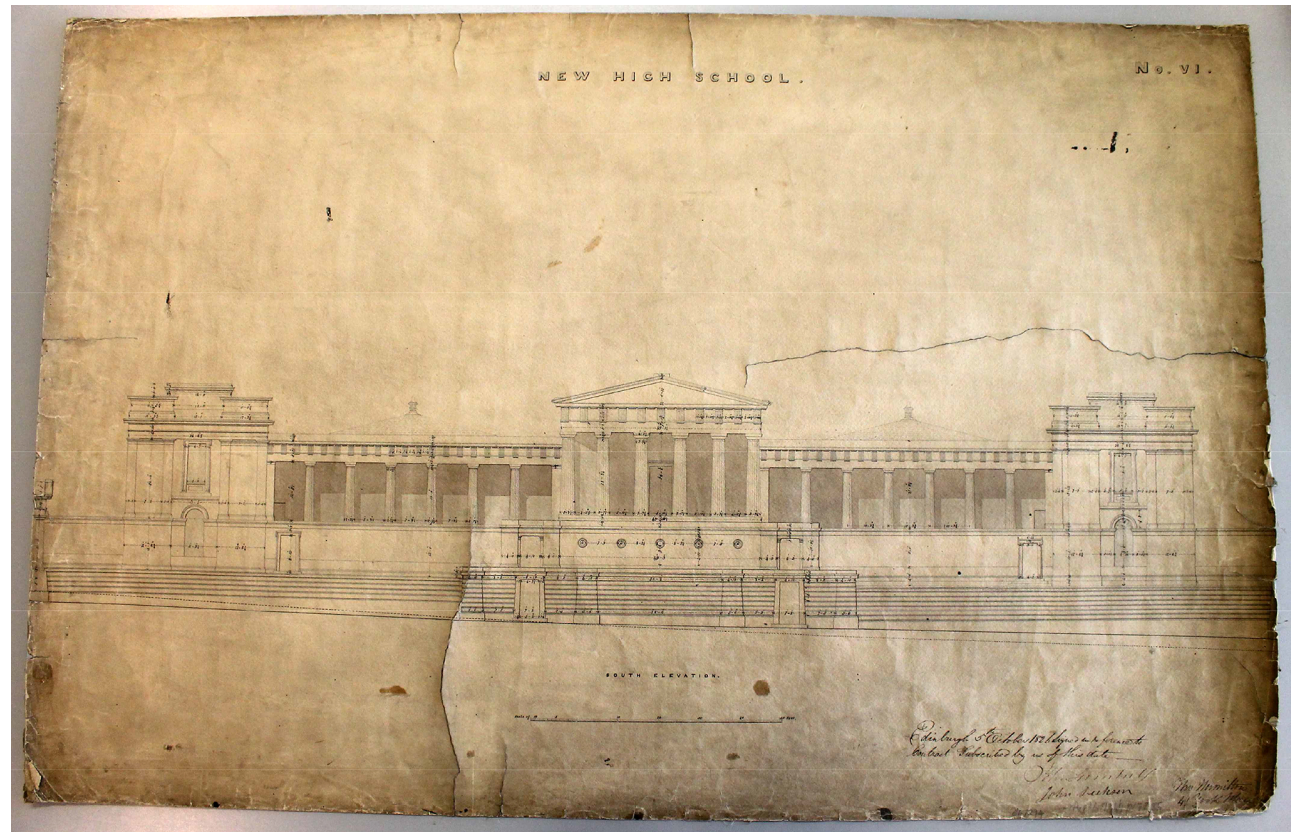

Historic Environment Scotland. 
Figure 12. - Grande salle de l'Old Royal High School en 1975.

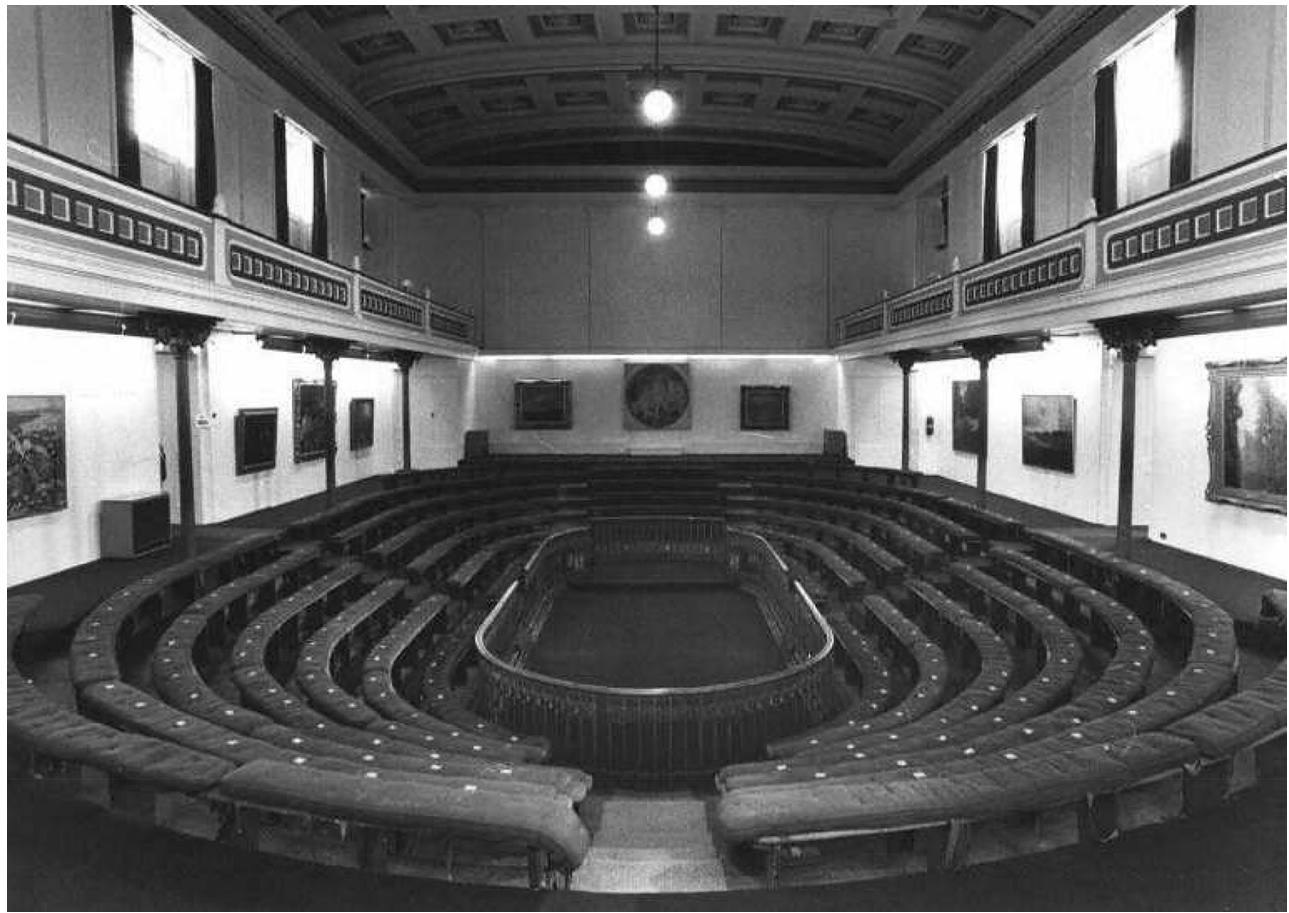

Historic Environment Scotland.

Figure 13. - Old Royal High School, Regent Terrace, côté sud-est.

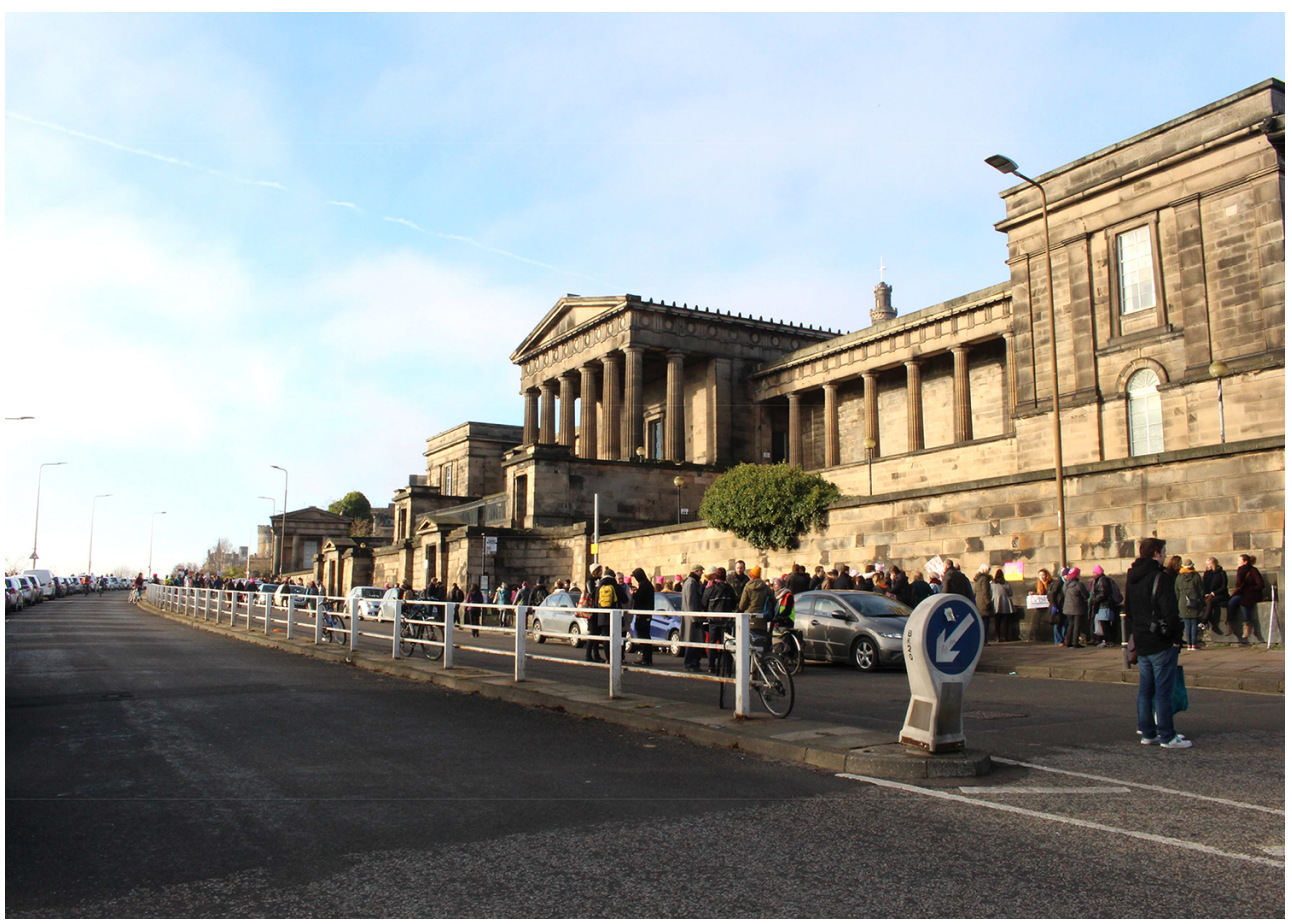

Photographie personnelle, mars 2017 
Figure 14. - « Say No to This », Royal High School Preservation Trust.
SAVE OLD ROYAL HIGH

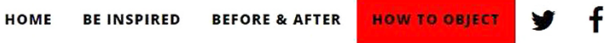

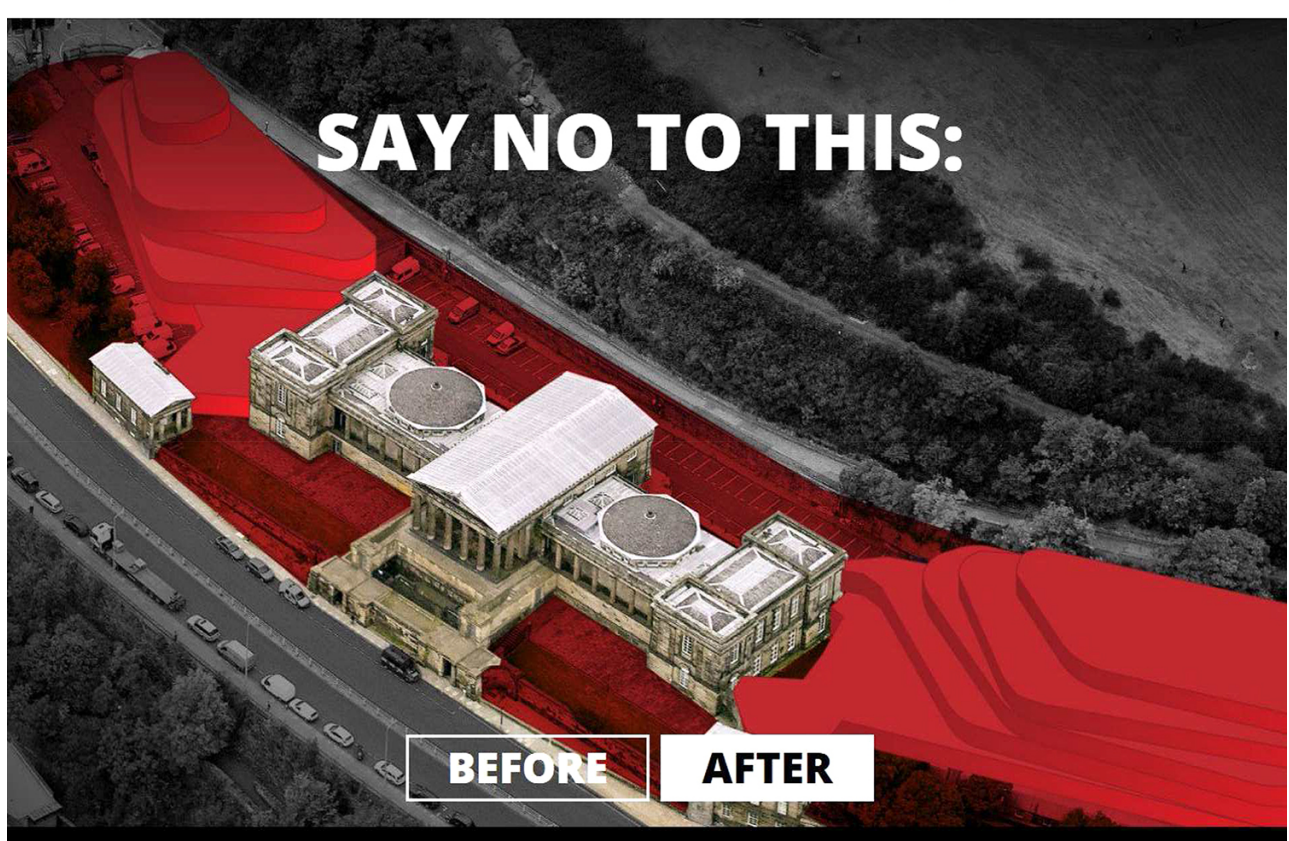

Disponible sur <www.rhspt.org> (consulté le 23 mars 2017).

\section{BIBLIOGRAPHIE}

\section{Sources primaires}

Address from the Town Council of Edinburgh on the Subject of the New Buildings for the High School of Which the Foundation was Laid on 28 July 1825, 1825, Édimbourg, s. n.

ARNOT Hugo, 1816, The History of Edinburgh, from the Earliest Accounts to the Year 1780 [...] To Which is Added a Sketch of the Improvements of the City, from 1780 to 1816. Embellished with an Elegant Plan of the City, Édimbourg, Thomas Turnbull.

Blackwood's Edinburgh Magazine, juillet 1819, vol. 5, n 28, p. 377-387.

CockBURn Henry, 1856, Memorial of His Time, New York, D. Appleton.

EDINBURGH CITY CoUnCIL, 2016, Neighbour Comment Support Submitted Online, 11 juillet. Disponible sur <http://citydev-portal.edinburgh.gov.uk/idoxpa-web/files/

F67AB52C9779249CFFBF929F38DCE1FB/pdf/15_05665_LBC-

NEIGHBOUR_COMMENT_SUPPORT_SUBMITTED_ONLINE-3382184.pdf> (consulté le 20 août 2017).

EdInBURGH City Council, 2017a, Decision, 27 février. Disponible sur <http://

citydevportal.edinburgh.gov.uk/idoxpaweb/files/52D1230D543AC2C396815407F17516CB/pdf/

15_05665_LBC--3552590.pdf> (consulté le 20 août 2017). 
EDINBURGH City CouncIL, 2017b, Minutes of the Planning Committee, Édimbourg, 30 mars.

Edinburgh Town Council Minutes, 29 janvier, 16 février 1577, 10 et 23 mai 1578.

Edinburgh Town Council Minutes, vol. XCV.

EdinBURGH World HeRITAge, 2005, The Old and New Towns of Edinburgh World Heritage Site, Management Plan, July 2005, Édimbourg.

EDINBURGH WORLD HERITAGE, 2011, The Old and New Towns of Edinburgh World Heritage Site Management Plan 2011-2016, Édimbourg.

EDINBURGH World HeRITAGE, 2017a, Lettre d'Adam Wilkinson à Jenny Bruce, Mairie d'Édimbourg, 23 mars.

EDINBURGH WORLD HERITAGe, 2017b, The Old and New Towns of Edinburgh World Heritage Site Draft Management Plan 2017-2022, Édimbourg, 30 mars.

EDINBURGH WORLD HERITAGE, 2017c, « Edinburgh to be part of major EU-funded historic cities project », 19 mai. Disponible sur <www.ewht.org.uk/news/607/102/Edinburgh-to-be-part-ofmajor-EU-funded-historic-cities-project> (consulté le 20 août 2017).

GRISCOM John, 1824, A Year in Europe [...], $2^{\mathrm{e}}$ éd., New York, Collins.

Historic ENVIRONMENT SCOTLAND, 2015a, lettre au Planning and Strategy Department Council of Edinburgh, 17 septembre. Disponible sur <www.historicenvironment.scot/media/3110/royalhigh-school-objection.pdf> (consulté le 20 août 2017).

Historic Environment SCotland, 2015b, lettre au Planning and Strategy Department Council of Edinburgh, 30 septembre. Disponible sur <www.historicenvironment.scot/media/3111/royalhigh-school-letter.pdf> (consulté le 20 août 2017).

Historic ENVIRONMENT SCOTLAND, 2016, The Old and New Towns of Edinburgh World Heritage Site, A Short Guide, Édimbourg, Historic Scotland.

HISTORIC SCOTLAND, 2007, Inventory of Gardens and Designed Landscapes in Scotland, Édimbourg, Historic Scotland.

Icomos, 2015, ICOMOS Technical Review, novembre.

KINCAID Alexander, 1787, The History of Edinburgh, from the Earliest Accounts to the Present Time, Édimbourg, N. R. Cheyne.

LAING David, 1817, This Volume Contains Extracts from the Records of the Town Council of Edinburgh, Relative to the High School of Edin. Interspersed with a Few Other Notices Connected with the Same. Taken in 1817 , s. $\mathrm{n}$.

Letter to the Right Hon. the Lord Provost of the City of Edinburgh Regarding the System of Education Pursued at the High School, 1829, Édimbourg, Adam Black.

LYNCH Peter, 2002, The History of the Scottish National Party, Cardiff, Welsh Academic Press.

PLAYFAIR William H., 1819, Report to the Right Honourable the Lord Provost, Magistrates, and Council of the City of Edinburgh [...] On a Plan for Laying out the New Town between Edinburgh and Leith, Etc., Édimbourg, Alex Smellie.

REID Peter, 1824, A Letter to the Patrons of the High School, and the Inhabitants of Edinburgh, on the Abuse of Classical Education, and on the Formation of a National School [...], Édimbourg, P. Brown. 
Royal High SCHool Preservation TRUSt, « A New Concert Hall ». Disponible sur <www.rhspt.org> (consulté le 20 août 2017).

Scots Magazine, juin 1777, vol. 39.

SCOTTISH GOVERNMENT, 2011, Scotland's Cities: Delivering for Scotland, décembre. Disponible sur <www.gov.scot/Resource/Doc/365367/0124252.pdf> (consulté le 20 août 2017).

ScotUS, 1822, A Letter to the Lord Provost, on the Mischievous Tendency of a Scheme for Abolishing the High School of Edinburgh, Édimbourg, Bell \& Bradfute.

STARK William, 1814, Report to the Right Honourable the Lord Provost, Magistrates, and Council of the City of Edinburgh, and the Governors of George Heriot's Hospital [...] On the Plans for Laying out the Grounds for Buildings between Edinburgh and Leith, Édimbourg, Printed by A. Smellie.

The Edinburgh Reporter, 17 décembre 2015. Disponible sur <www.theedinburghreporter.co.uk/ 2015/12/councillors-reject-luxury-hotel-plans-for-former-royal-high-school/> (consulté le 20 août 2017).

The Scotsman, 14 janvier 1826.

UNESCO, 1995, World Heritage List, Old and New Towns of Edinburgh. Disponible sur <http:// whc.unesco.org/en/list/728> (consulté le 20 août 2017).

UNESCO, 1996, UNESCO Convention Concerning the Protection of the World Cultural and Natural Heritage, World Heritage Committee, 9th Session Berlin, 4-9 décembre 1995, rapport du 31 janvier. Disponible sur <http://whc.unesco.org/en/list/728/documents/> (consulté le 20 août 2017).

UNESCO, 2009, UNESCO Convention Concerning the Protection of the World Heritage Committee, Decisions Adopted at the 32nd Session of the World Heritage Committee, Quebec City 2008, 31 mars. Disponible sur <http://whc.unesco.org/archive/2008/whc08-32com-24reve.pdf> (consulté le 20 août 2017).

UNESCO, 2011, Decisions Adopted at the 35th Session of the World Heritage Committee, Paris June 2011, 7 juillet. Disponible sur <http://whc.unesco.org/archive/2011/whc11-35com-20e.pdf> (consulté le 20 août 2017).

UNESCO, 2012, Operational Guidelines for the Implementation of the World Heritage Convention, juillet. Disponible sur <http://whc.unesco.org/archive/opguide12-en.pdf> (consulté le 20 août 2017).

UNESCO, 2015, Lettre de Mechtild Rossler à Hannah Jones, Department for Culture, Media and Sport, London, 18 décembre.

WILSON Daniel, 1848, Memorials of Edinburgh in the Olden Time, vol. 2, Édimbourg, Thomas C. Jack. WooD John, 1823, Plan of the City of Edinburgh, Including All the Latest and Intended Improvements, Édimbourg, T. Brown.

\section{Sources secondaires}

ANDERSON, Robert D., 1983, Education and Opportunity in Victorian Scotland, Schools and Universities, Édimbourg, Edinburgh University Press.

BARCLAY John B., 1974, The Tounis Scule, The Royal High School of Edinburgh, Édimbourg, Royal High School Club.

BLACK David, 2001, All the First Minister's Men: Uncovering the Truth behind the Holyrood Scandal, Édimbourg, Birlinn.

Buchanan Hugh, 2015, « Classic Revival or Greek Tragedy? », Country Life, 6 mai, p. 26-27. 
CALDERWOOD David, 1842, The History of the Kirk of Scotland, vol. 5, Édimbourg, Wodrow Society.

Colvin Howard M., 1995, A Biographical Dictionary of British Architecture, 1600-1840, $3^{\text {e éd., }}$ New Haven \& London, Yale University Press.

DAVIE George E., 1961, The Democratic Intellect, Scotland and Her Universities in the Nineteenth Century, Édimbourg, Edinburgh University Press.

GIFFORD John, 2014, « The National Monument of Scotland », Architectural Heritage, vol. 25, $\mathrm{n}^{\circ}$ 1, p. 43-83.

GIFFORD John, MCWILLIAM Colin \& WALKER David (éds), 1984, The Buildings of Scotland, Edinburgh, Harmondsworth, Penguin.

Glendinning Miles, Macinnes Ranald \& MACKeChnie Aonghus (éds), 1996, A History of Scottish Architecture: From the Renaissance to the Present Day, Édimbourg, Edinburgh University Press.

HARRIS George, 2000, « Enlightenment and Empire. The Foundation of the Edinburgh Academy », The Academical, $\mathrm{n}^{\circ} 9$.

LOWREY John, 2001, " From Caesarea to Athens, Greek Revival Edinburgh and the Question of Scottish Identity within the Unionist State ", Journal of the Society of Architectural Historians, vol. 60, $\mathrm{n}^{\circ}$ 2, p. 136-157.

MCKEAN Charles, 2005, « Twinning Cities: Modernisation Versus Improvement », dans B. Edwards et P. Jenkins (éds), Edinburgh, The Making of a Capital City, Édimbourg, Edinburgh University Press, p. 42-63.

MURRAY John, 1997, A History of the Royal High School, Édimbourg, Royal High School.

Ross William C. A., 1934, The High School of Edinburgh, Édimbourg, Oliver \& Boyd.

STEVEN William, 1849, The History of the High School of Edinburgh, Édimbourg, Maclachlan \& Stewart. SUMMERSON John, 1953, Architecture in Britain, 1530-1830, Londres, Penguin.

TROTTER James J., 1911, The Royal High School, Edinburgh, Londres, Sir I. Pitman.

WALKER David (éd.), 1984, Scottish Pioneers of the Greek Revival, Édimbourg, Scottish Georgian Society.

WALKER David, 1989, St Andrew's House: An Edinburgh Controversy, 1912-1939, Édimbourg, Historic Scotland.

WALKER David, s. d., Dictionary of Scottish Architects, 1660-1980. Disponible sur $<$ www.scottisharchitects.org.uk/architect_full.php?id=200136>.

WATTERS Diane, 2014, « The Beautiful and the Good: Classical School Architecture and Educational Elitism in Early Nineteenth-Century Edinburgh », Architectural History, vol. 57, p. 277-321.

\section{NOTES}

1. Les quatre premiers historiens de la «Schola Regia» sont William Steven (1849), James J. Trotter (1911), William C. A. Ross (1934) et John B. Barclay (1974).

2. Cette obligation est renouvelée par la municipalité en 1584 (Barclay, 1974, p. 8).

3. Edinburgh Town Council Minutes, 29 janvier et 16 février 1577, 10 et 23 mai 1578.

4. Ce blason figure actuellement dans la grande salle de la Royal High School sur Regent Road et une copie moulée est présente depuis 1973 dans les locaux de la nouvelle l'école de Barnton. Si le 
choix de cette devise demeure obscur, il est affirmé qu'un État ne saurait prospérer sans système éducatif reposant sur un solide enseignement humaniste.

5. En 1590, Hercules Rollock participe aux cérémonies officielles organisées lors de l'entrée à Édimbourg de la reine Anne de Danemark, nouvelle épouse de Jacques VI.

6. Le projet de construction est financé par la ville et par une souscription publique: "The subscribers received from the managers of the Royal Infirmary a piece of ground from their garden for enlarging the present area, and had reason to hope that the Incorporation of Surgeons would, for the same purpose, yield them a small piece of ground from their garden adjoining [...]. » (Edinburgh Town Council Minutes, vol. XCV, p. 94-97) Deux pamphlets sont publiés en 1775 : « Proposals for a new Grammar in Edinburgh » et «Considerations on the Proposals for Building a New Grammar School in the City of Edinburgh; setting forth the inutility of such a scheme, and the prejudice that may result therefrom to education. With a Proposal for the Increase of Latin Schools, and some Observations on the present mode of Teaching " (Steven, 1849, p. 122-123). La pose de la première pierre, le 24 juin 1777, prend la forme d'une cérémonie grandiose présidée par le banquier et Grand Maître d'Écosse Sir William Forbes of Pitsligo. Cette construction est largement commentée par la presse de l'époque (Scots Magazine, juin 1777, vol.39, p. 332-334; consulter aussi Ruddiman's Weekly Magazine, Edinburgh Evening Courant, Caledonian Mercury).

7. L'école de Laing se compose, au rez-de-chaussée, d'une grande salle, d'une bibliothèque et d'un salon et, à l'étage, de cinq salles de classe (Kincaid, 1787, p. 187-189).

8. Citons James Hutton, John Watson, Robert Adam, Alexander Wedderburn, Robert Mylne, Thomas Coutts, Henry Dundas, premier vicomte Melville, et William Smellie.

9. Address from the Town Council of Edinburgh on the Subject of the New Buildings for the High School of Which the Foundation was Laid on 28 July 1825, 1825, Édimbourg, s. n., p. 1.

10. À son retour à New York, Griscom crée une institution fortement inspirée de la High School (celle-ci disparaît en 1832).

11. L'effectif est de 409 garçons en 1777 et de 629 en 1809.

12. Le plan de James Craig (1768) pour ce qui allait devenir la première ville nouvelle remporte le concours organisé par la municipalité.

13. Address from the Town Council of Edinburgh on the Subject of the New Buildings for the High School of Which the Foundation was Laid on 28 July 1825, 1825, Édimbourg, s. n., p. 4.

14. Des voix critiques craignent alors que l'Academy ne fasse concurrence à la Royal High School: "The present High School is too remote from many parts of the city, particularly the New Town. It might even be conceded [...] that some of the classes are too much crowded; but it will not follow from all this, that another school, an opposition, a rival school, should be established on the ruins of the present seminary. " (Scotus, 1822, p. 2)

15. Pilier de l'Academy, Horner est aussi le fondateur de l'Edinburgh School of Arts, ancêtre de l'université Heriot-Watt, qui voit le jour en 1821. Cette institution s'adresse aux enfants d'artisans.

16. " [...] one day on the top of one of the Pentlands, emblematic of the solidity of our foundation and of the extent of our prospects, we two [Leonard Horner and myself] resolved to set about the establishment of a new School. On taking others into council, we found that the conviction of the inadequacy of the High School was far more general than we supposed. » (Cockburn, 1856, p. 390-391) Le soutien du Tory John Russell fut un atout pour l'Academy.

17. Scotus $(1822$, p. 6). Il est rappelé que le système éducatif de la Royal High School est inclusif, construit sans préjugé de classe ni de fortune, et qu'il a pu former des générations d'enfants de tous milieux et favoriser l'émergence de talents pour le plus grand succès de la nation et de l'Empire.

18. Davie relie la perte d'une tradition écossaise en matière éducative à la crise intellectuelle et culturelle qui affecte l'Écosse au XIXe siècle. 
19. En 1827, au terme d'un débat houleux, la prononciation anglaise du latin s'impose à l'Academy. Pour une analyse du système éducatif écossais, son ancienneté et excellence, se reporter à l'ouvrage de George Davie et à la critique de Robert Anderson.

20. Cet édifice est conçu autour d'une grande salle ovale à l'arrière de laquelle se situe le bureau du directeur. Quatre salles de cours et quatre pièces attenantes figurent de part et d'autre de la grande salle centrale. William Burn est d'abord formé à Londres par l'architecte néo-grec Sir Robert Smirke, auteur du British Museum, avant de s'installer en Écosse et d'y remporter de multiples commandes (cf. <www.scottisharchitects.org.uk/architect_full.php?id=200136>).

21. The Scotsman, 14 janvier 1824.

22. L'architecte Thomas Hamilton soumet des plans pour ce site actuellement siège de la Royal Bank of Scotland, en mai 1824. Ces plans sont conservés dans la bibliothèque de l'école à Barnton. L'Excise Office est vendue à la Royal Bank of Scotland en mars 1825, ce qui signifie l'abandon du choix de ce site pour l'école.

23. Ses aquarelles Views of Greece sont exposées en 1822 à Édimbourg. Select Views of Greece (1824-1826) inclut des eaux fortes représentant des vues du Parthénon, de l'Acropole et du temple de l'Érechthéion à Athènes.

24. Blackwood's Edinburgh Magazine, juillet 1819, vol. 5, $\mathrm{n}^{\circ} 28$, p. 377-387.

25. "[...] there cannot be a doubt that the whole surface of the Earthen Mound, and North Loch to the eastward of it, would be employed in a manner much more useful and creditable to the city, were it devoted wholly to the accommodation of the most important academy in Scotland, than it is at present, a mere depot of menageries for wild beasts, the resort of washerwomen, and the scene of everything that is vulgar and offensive. » (Scotus, 1822, p. 23)

26. Le site du Calton Hill est propriété de la ville, ce qui épargne à la municipalité l'achat d'un terrain.

27. "The inspiration to break with precedent, by responding to the landscape rather than dominating it, came from William Stark [...] Stark's recommendations [...] elevated the values of landscape, contour, prospect and trees above the seduction of geometry. » (McKean, 2005, p. 55)

28. "I have also [...] introduced large public gardens, trusting that by judicious and careful planting, an assemblage of trees and buildings may be obtained, without which architecture is deprived of half its beauty. » (Playfair, 1819, p. 5)

29. Le changement d'esthétique s'exprime dans cette longue description de la Royal High : « [...] and we trust that the time has forever gone by, when the beauty of a street shall be identified with its interminable length in a straight line and a most Presbyterian equality of elevation without a single pillar or projection to relieve or enliven the boundless mass. » (The Scotsman, 14 janvier 1826, p. 31)

30. Address from the Town Council of Edinburgh on the Subject of the New Buildings for the High School of Which the Foundation was Laid on 28 July 1825, 1825, Édimbourg, s. n.

31. L'entrée de l'école se situait côté nord. L'entrée du portique, côté sud, n'était utilisée que pour de rares occasions, en particulier lors de la remise des diplômes en fin d'année.

32. The Scotsman, 14 janvier 1826, p. 31.

33. "It was about this time that the foolish phrase, "The Modern Athens," began to be applied to the capital of Scotland; a sarcasm, or a piece of affected flattery, when used in a moral sense ; but just enough if meant only as a comparison of the physical features of the two places. ». (Cockburn, 1856, p. 274)

34. L'origine de l'expression fait débat.

35. Plusieurs manifestations furent alors organisées par Margo MacDonald.

36. Voir aussi la lettre d'objection du 17 septembre 2015 adressée par Historic Environment Scotland au Planning and Strategy Department de la mairie d'Édimbourg.

37. Critère 2 de l'UNESCO: «Témoigner d'un échange d'influences considérable pendant une période donnée ou dans une aire culturelle déterminée, sur le développement de l'architecture ou de la technologie, des arts monumentaux, de la planification des villes ou de la création de paysages. " Critère $4:$ "Offrir un exemple éminent d'un type de construction ou d'ensemble 
architectural ou technologique ou de paysage illustrant une ou des périodes significative(s) de l'histoire humaine. " (UNESCO, Operational Guidelines for the Implementation of the World Heritage Convention, juillet 2012, p. 20-21)

38. UNESCO, UNESCO Convention Concerning the Protection of the World Cultural and Natural Heritage, World Heritage Committee, 9th Session Berlin, 4-9 décembre 1995, rapport du 31 janvier 1996, p. 49. Pour une carte de la surface du site classé (2005), voir <http://whc.unesco.org/en/list/728/ documents/> ou Historic Environment Scotland, The Old and New Towns of Edinburgh World Heritage Site, A Short Guide, 2016, p. 3.

39. UNESCO, World Heritage List, Old and New Towns of Edinburgh, 1995, p. 78.

40. Edinburgh World Heritage, The Old and New Towns of Edinburgh World Heritage Site, Management Plan, July 2005, 2005, p. 28.

41. Soigneusement aménagé lors des constructions de la Royal High School, de Royal Terrace et de Regent Terrace, le versant sud du Calton Hill est respectueux des idées de Stark et de Playfair. Aucune construction ne devait figurer au-dessus de l'école pour préserver la topographie de la colline.

42. Lorsque St Andrew's House fut construite, dans le cadre d'une dévolution administrative accrue, l'architecte avait pleinement intégré l'importance de la Royal High à proximité (Walker, 1989, p. 8-19).

43. Créé en 1999 par la mairie d'Édimbourg et Historic Scotland, Edinburgh World Heritage Trust (EWHT) est issu de la fusion entre Old Town Renewal Trust et New Town Conservation Committee. Il garantit la préservation de la Vieille Ville et des Nouvelles Villes d'Édimbourg, sites classés au patrimoine mondial de l'UNESCO, et conseille la municipalité dans ses projets d'urbanisme. EWHT offre également des recommandations aux particuliers et aux entreprises.

44. «Councillors reject luxury hotel plans for former Royal High School », The Edinburgh Reporter, 17 décembre 2015.

45. Lettre de Mechtild Rossler (UNESCO) à Hannah Jones (Department for Culture, Media and Sport), 18 décembre 2015.

46. En tant qu'État partie à la Convention pour la sauvegarde du patrimoine mondial depuis 1972, le Royaume-Uni doit répondre, auprès de l'UNESCO, de l'état de conservation de ses sites classés.

47. ICOMOS-UK rend compte auprès de l'UNESCO des changements opérés sur les sites classés au patrimoine mondial. Le rapport ICOMOS a été transmis à la mairie d'Édimbourg et à Historic Environment Scotland (HES) via le UK Government Department for Culture, Media and Sport et le gouvernement écossais (Culture and Heritage Directorate). Une réponse du gouvernement écossais, sur la base des informations fournies par la mairie et HES, est en préparation. Ce support permettra au Department for Culture, Media and Sport de rédiger une réponse officielle. Cette procédure complexe est destinée à ce que les sites inscrits sur la liste du patrimoine mondial satisfassent pleinement à leurs obligations au regard de la Convention du patrimoine mondial. Sur un total de plus de 1000 sites classés au patrimoine mondial, 29 sont situés au Royaume-Uni et 6 en Écosse (les Ancienne et Nouvelle Villes d'Édimbourg, New Lanark, le site néolithique de l'île d'Orkney, Saint Kilda, le mur d'Antonin et le Forth Bridge).

48. En 2008, l'UNESCO effectuait une mise en garde concernant la démolition et la transformation du site de Caltongate, en plein cœur de la Vieille Ville (UNESCO, UNESCO Convention Concerning the Protection of the World Heritage Committee, Decisions Adopted at the 32nd Session of the World Heritage Committee, Quebec City 2008, 31 mars 2009, p. 136-137 ; UNESCO, Decisions Adopted at the 35th Session of the World Heritage Committee, Paris June 2011, 7 juillet 2011. Le Ribbon Hotel, dans le St James Centre, a attiré l'attention des médias. Il est à craindre que cette construction imposante et futuriste articulée autour d'une structure hélicoïdale en métal s'insèrera mal dans ce site classé (ICOMOS Technical Review, novembre 2015, p. 2).

49. Le gouvernement écossais pourra ensuite choisir, ou non, de suivre l'avis consultatif des rapporteurs du DPEA. 
50. Les permis sont en général valables pour trois ans mais, dans le cas présent et en raison de l'offre concurrente, l'autorisation de la mairie a été accordée pour une durée de sept ans (Edinburgh City Council, Decision, 27 février 2017)

51. Le projet pour l'école de musique est disponible à l'adresse suivante : <www.rhspt.org>. Le RHSPT a été créé en avril 2015 pour tenter de trouver une destination viable et pérenne au bâtiment de l'ancienne Royal High School.

52. Edinburgh City Council, Neighbour Comment Support Submitted Online, 11 juillet 2016.

53. "The changes that have resulted in this new application do not come close to addressing our concerns and objections to the earlier scheme. " (Edinburgh World Heritage Trust, Lettre d'Adam Wilkinson à Jenny Bruce, Mairie d'Édimbourg, 23 mars 2017, p. 1)

54. La première feuille de route pour ce site classé au patrimoine mondial a couvert la période 2005-2010. La seconde feuille de route est arrivée à échéance en 2016.

55. Edinburgh City Council, Minutes of the Planning Committee, 30 mars 2017, p. 1-2. Edinburgh World Heritage, The Old and New Towns of Edinburgh World Heritage Site Draft Management Plan 20172022, 30 mars 2017.

56. Scottish Government, Scotland's Cities: Delivering for Scotland, décembre 2011, p. 33.

57. Edinburgh World Heritage Trust, «Edinburgh to be part of major EU-funded historic cities project », 19 mai 2017.

\section{RÉSUMÉS}

$\mathrm{Au}$ début du XIX ${ }^{\mathrm{e}}$ siècle, Édimbourg s'affirme comme la capitale intellectuelle et culturelle du Royaume-Uni. L'ancienne Royal High School, bâtiment de style néo-grec construit entre 1825 et 1829 par l'architecte Thomas Hamilton (1784-1858), incarne l'ambition de la ville de devenir nouvelle Athènes. Construite sur le flanc sud du Calton Hill, un site préservé face à la Vieille Ville, l'école s'insère dans un paysage pittoresque et symbolique qui fait écho à celui de l'Acropole grecque. Les Ancienne et Nouvelle Villes furent classées au patrimoine mondial de l'UNESCO en 1995 et la valeur universelle du site était à cette occasion consacrée. Ce statut est pourtant menacé par plusieurs projets de développement urbain. La transformation de l'ancienne Royal High School est au cœur des enjeux actuels liés à la conservation des monuments historiques.

At the beginning of the nineteenth century, Edinburgh's ambition was to become the intellectual and cultural capital of the United Kingdom. The Old Royal High School, a building in the Greek style erected between 1825 and 1829 by architect Thomas Hamilton (1784-1858), expresses the city's ambition to become another Athens. On the south side of the Calton Hill, the school faces the old Town and exploits a picturesque, symbolic site which recalls the Greek Acropolis. The universal value of the site was acknowledged in 1995 when the Old and New Towns of Edinburgh became a World Heritage Site. Such a status is now potentially at risk, due to the many redevelopment schemes in the city. The current project for the old Royal High School fully illustrates the tensions over the preservation of historic monuments. 
INDEX

Mots-clés : Édimbourg, Old Royal High School, xixe siècle, conservation des monuments historiques, patrimoine mondial

Keywords : Edinburgh, Old Royal High School, nineteenth century, preservation of historic monuments, World Heritage Site

\section{AUTEUR}

\section{CLARISSE GODARD DESMAREST}

Université de Picardie Jules Verne / Institut universitaire de France.

Clarisse Godard Desmarest est agrégée d'anglais, diplômée de Sciences Po Paris et docteur de l'université Paris-Sorbonne. Maître de conférences en civilisation britannique à l'université de Picardie Jules Verne (Amiens) et membre de l'Institut universitaire de France, ses travaux de recherche portent sur l'Écosse aux XVII ${ }^{\mathrm{e}}$ et XVIII ${ }^{\mathrm{e}}$ siècles : l'architecture, les collections, les parcs et jardins. 